ESAIM: COCV 18 (2012) 987-1004

DOI: $10.1051 / \mathrm{cocv} / 2011201$
ESAIM: Control, Optimisation and Calculus of Variations

www.esaim-cocv.org

\title{
LINEAR-QUADRATIC OPTIMAL CONTROL FOR THE OSEEN EQUATIONS WITH STABILIZED FINITE ELEMENTS
}

\author{
Malte Braack ${ }^{1}$ and Benjamin Tews ${ }^{1}$
}

\begin{abstract}
For robust discretizations of the Navier-Stokes equations with small viscosity, standard Galerkin schemes have to be augmented by stabilization terms due to the indefinite convective terms and due to a possible lost of a discrete inf-sup condition. For optimal control problems for fluids such stabilization have in general an undesired effect in the sense that optimization and discretization do not commute. This is the case for the combination of streamline upwind Petrov-Galerkin (SUPG) and pressure stabilized Petrov-Galerkin (PSPG). In this work we study the effect of different stabilized finite element methods to distributed control problems governed by singular perturbed Oseen equations. In particular, we address the question whether a possible commutation error in optimal control problems lead to a decline of convergence order. Therefore, we give a priori estimates for SUPG/PSPG. In a numerical study for a flow with boundary layers, we illustrate to which extend the commutation error affects the accuracy.
\end{abstract}

Mathematics Subject Classification. 35A15, 49Mxx, 65G99, 65M60, 76D05, 76D07, $76 \mathrm{D} 55$.

Received November 26, 2010. Revised September 15, 2011.

Published online 16 January 2012.

\section{INTRODUCTION}

The Oseen linearization is the most popular linearization of the Navier-Stokes equations and is used for mathematical analysis and for the design of numerical solution schemes as well. In the case of dominant convection, the convective parts must be treated appropriately in order to obtain a stable discrete scheme. Among the most popular finite element stabilization is the streamline upwind Petrov-Galerkin (SUPG) [13] method. In the case of not inf-sup stable elements, as e.g. equal-order elements for pressure and velocity, a further stabilization is needed, e.g. the pressure stabilized Petrov-Galerkin (PSPG) [7] method. Such SUPG/PSPG stabilization techniques cause certain difficulties in the context of optimal control problems.

This is due to the fact that the optimal control problem involves another PDE, the so called adjoint equation, which is also of Oseen type and need to be stabilized. If one firstly discretizes the state equation and then build up the optimality system, called discretize-optimize approach, the stabilization scheme of the adjoint equation depends on the stabilization scheme applied to the state equation. Therefore this equation may not be stabilized in an appropriate way. Due to the optimality conditions the adjoint state is strongly associated with the control

\footnotetext{
Keywords and phrases. Oseen, Navier-Stokes, optimal control, finite elements, stabilized methods.

1 Mathematisches Seminar, Christian-Albrechts-Universität zu Kiel, Ludewig-Meyn-Str. 4, 24098 Kiel, Germany.

braack@math.uni-kiel.de; tews@math.uni-kiel.de
} 
and a potentially lack of accuracy of the adjoint state carries over to the control. Alternatively, one may choose the optimize-discretize approach, which means that the optimality system is built up first on the continuous level. Afterwards, the state and adjoint equation are discretized separately. Whereas the continuous optimality system is symmetric by definition, the drawback of this technique reflects in possible non-symmetry of the resulting discrete optimality system. Consequently, the computed control may be significantly affected by the way of defining the discrete optimality condition. In contrast to this, for symmetric stabilization schemes optimizediscretize and discretize-optimize are equivalent and lead to optimal order of convergence [4]. In this work, we analyze the two versions of a classical non-symmetric discretization of the Oseen problem in the context of optimal control. Moreover, we compute the convergence order numerically and make comparisons with a symmetric scheme.

The discretization with stabilized finite elements of optimal control problems governed by scalar elliptic problems is an active field of research. An analytical error estimate for scalar linear elliptic equations with the SUPG method is given by Collis and Heinkenschloss in [8] where for the two approaches "discretize-optimize" and "optimize-discretize" different a priori estimates are derived. Moreover, local estimates have been published recently in [11]. An error estimate for a particular symmetric finite element method (local projection) has been carried out by Becker and Vexler [3] for such scalar linear elliptic equations. The paper by Lube and Tews [16] studies the local projection stabilization applied to quadratic optimal control problems governed by convection diffusion equations with and without control constraints. The interior penalty method for optimal control is analyzed recently by Hinze et al. [12] and Yan and Zhou [19]. As a reference for the streamline diffusion method we like to refer to [18]. An a posteriori estimator for scalar advection-diffusion problems has been published by Dede and Quarteroni [9].

For the Oseen system the upper mentioned effect is still poorly analyzed and numerical investigations are rare, e.g. Abraham et al. [1] numerically observed significant effects in view of choosing the stabilization constants. In this work, we try to close this gap. We give an a priori error analysis of the optimal control problem with the Oseen system and present numerical results. Furthermore, we give a comparison with a symmetric scheme [6].

The Oseen equations in a polyhedral domain $\Omega \subset \mathbb{R}^{d}, d \in\{2,3\}$ with Lipschitz boundary $\partial \Omega$ are formulated for the pressure $p$ and the velocity field $\mathbf{v}$ by

$$
\begin{aligned}
-\mu \Delta \mathbf{v}+(\mathbf{b} \cdot \nabla) \mathbf{v}+\sigma \mathbf{v}+\nabla p+\mathbf{u}=\mathbf{f} & & \text { in } \Omega \\
\nabla \cdot \mathbf{v}=0 & & \text { in } \Omega \\
\mathbf{v}=0 & & \text { on } \partial \Omega,
\end{aligned}
$$

where $\mathbf{f}$ stands for given data, $\mu>0, \sigma \geq 0$ and $\mathbf{b}$ is a divergence free vector field, $\nabla \cdot \mathbf{b}=0$. Furthermore, $\mathbf{u}$ is a control function which has to be determined such that a functional $J$ becomes minimal:

$$
J(\mathbf{v}, \mathbf{u}):=\frac{1}{2}\left\|\mathbf{v}-\mathbf{v}_{d}\right\|_{0}^{2}+\frac{\alpha}{2}\|\mathbf{u}\|_{0}^{2} \rightarrow \min .
$$

Here, $\mathbf{v}_{d}$ is a target velocity field, and $\alpha>0$ is a given positive constant.

The content of this work is structured as follows. We start in Section 2 with the variational formulation of the optimal control Oseen system (1.1)-(1.2). The discretization by finite elements and the formulation of the finite-dimensional linear systems for SUPG/PSPG and for local projection stabilization (LPS) is given in Section 3. In Section 4 we give an a priori analysis. In the last section we report on numerical results of a model problem with an exponential boundary layer.

\section{VARIATIONAL FORMUlation OF THE OPTIMIZATION PROBLEM}

\subsection{Notations}

We use the standard notation $L^{2}(\Omega)$ for Lebesgue integrable functions and $H^{r}(\Omega)$ for the Sobolev space with derivatives of order $r \geq 0$ (see e.g. [10]). We will use a context-sensitive notation for the $L^{2}$-inner product applied 
to scalar, vector-valued and tensor-valued functions. Let $\omega \subset \Omega$ a measurable subset of the domain $\Omega$. Then, for scalar functions $p, q \in L^{2}(\Omega)$, we denote by $(p, q)_{\omega}$ the usual inner product in $L^{2}(\omega)$. If $\mathbf{v}, \mathbf{w}: \Omega \rightarrow \mathbb{R}^{d}$ are vector-valued functions with the components $v_{i}, w_{i} \in L^{2}(\Omega)$ and $A, B: \Omega \rightarrow \mathbb{R}^{d \times d}$ are matrix-valued functions with components $A_{i j}, B_{i j} \in L^{2}(\Omega)$ we define

$$
(\mathbf{v}, \mathbf{w})_{\omega}:=\sum_{i=1}^{d}\left(v_{i}, w_{i}\right)_{\omega} \quad \text { and } \quad(A, B)_{\omega}:=\sum_{i, j=1}^{d}\left(A_{i j}, B_{i j}\right)_{\omega} .
$$

This context-sensitive $L^{2}$-inner product induces the corresponding context-sensitive $L^{2}$-norm notation $\|\cdot\|_{0, \omega}$ and $H^{1}$-semi-norm or norm notation $|v|_{1, \omega}^{2}:=(\nabla v, \nabla v)_{\omega}$ or $\|v\|_{1, \omega}^{2}:=|v|_{1, \omega}^{2}+\|v\|_{0, \omega}^{2}$. In the case $\omega=\Omega$, we will omit the index $\omega$ and write simply $(\cdot, \cdot)$ and $\|\cdot\|_{r}$ or $|\cdot|_{r}$ for functions in $H^{r}(\Omega)^{n}$ where $n \in\{1, d\}$ and $H^{0}$ is formally identified with $L^{2}$. The natural weak solution space for the velocity $\mathbf{v}$ is

$$
H_{0}^{1}(\Omega)^{d}=\left\{\mathbf{v} \in H^{1}(\Omega)^{d}: \mathbf{v}=\mathbf{0} \text { a.e. on } \partial \Omega\right\} .
$$

The pressure space $L_{0}^{2}(\Omega)$ is the subspace of the $L^{2}(\Omega)$ functions with zero integral mean value. By y we denote the vector of velocities and pressure, $\mathbf{y}=(\mathbf{v}, p)$. Hence, $\mathbf{y}$ is sought in the Hilbert space $X:=H_{0}^{1}(\Omega)^{d} \times L_{0}^{2}(\Omega)$ and the control in a further Hilbert space, $\mathbf{u} \in Q \subset L^{2}(\Omega)^{d}$. By $\|\cdot\|_{0}$ and $(\cdot, \cdot)$ we denote the $L^{2}(\Omega)$-norm and $L^{2}(\Omega)$-scalar product, respectively. For a Hilbert space $H$ and its dual space $H^{*}$ the duality product between functions $g \in H$ and $f \in H^{\prime}$ is denoted

$$
\langle f, g\rangle=f(g) .
$$

\subsection{Variational formulation of the state equation}

In order to express the variational formulation of the state equation (1.1) we introduce the bilinear forms for the pair of test functions $\varphi=(\phi, \xi) \in X$

$$
\begin{aligned}
& A(\mathbf{y}, \boldsymbol{\varphi}):=(\nabla \cdot \mathbf{v}, \xi)+(\sigma \mathbf{v}, \boldsymbol{\phi})+((\mathbf{b} \cdot \nabla) \mathbf{v}, \phi)+(\mu \nabla \mathbf{v}, \nabla \boldsymbol{\phi})-(p, \nabla \cdot \phi) \\
& B(\mathbf{u}, \boldsymbol{\phi}):=(\mathbf{u}, \boldsymbol{\phi}) .
\end{aligned}
$$

For a right hand side $\mathbf{f} \in H^{-1}(\Omega)^{d}$ the weak formulation reads now: Seek the pair $\mathbf{y}=(\mathbf{v}, p) \in X$ s.t:

$$
A(\mathbf{y}, \boldsymbol{\varphi})+B(\mathbf{u}, \phi)=\langle\mathbf{f}, \phi\rangle \quad \forall \boldsymbol{\varphi}=(\phi, \xi) \in \mathbf{X} .
$$

\subsection{First and second order optimality system}

As usual for optimization problems, we define the Lagrangian functional $\mathcal{L}: X \times Q \times X \rightarrow \mathbb{R}$ with Lagrange multiplier $\mathbf{z}=\left(\mathbf{z}^{v}, z^{p}\right) \in X$,

$$
\mathcal{L}(\mathbf{y}, \mathbf{u}, \mathbf{z}):=J(\mathbf{y}, \mathbf{u})-A(\mathbf{y}, \mathbf{z})-B\left(\mathbf{u}, \mathbf{z}^{v}\right)+\left\langle\mathbf{f}, \mathbf{z}^{\mathbf{v}}\right\rangle .
$$

The necessary and, due to convexity, also sufficient first order optimality conditions for minimize the unconstraint functional $J$ are formulated in terms of the Frechet-derivatives with respect to the variables. The derivative with respect to the Lagrange multiplier leads to the state equation (2.2). The derivative with respect to the state leads to the so called adjoint equation,

$$
\partial_{y} \mathcal{L}(\mathbf{y}, \mathbf{u}, \mathbf{z})(\boldsymbol{\psi}) \equiv 0 \quad \Rightarrow \quad A(\boldsymbol{\psi}, \mathbf{z})=\left(\mathbf{v}-\mathbf{v}_{d}, \boldsymbol{\psi}^{v}\right) \quad \forall \boldsymbol{\psi}=\left(\boldsymbol{\psi}^{v}, \psi^{p}\right) \in X
$$

The derivative with respect to the control leads to the gradient equation,

$$
\partial_{u} \mathcal{L}(\mathbf{y}, \mathbf{u}, \mathbf{z})(\boldsymbol{\lambda}) \equiv 0 \quad \Rightarrow \quad \alpha(\mathbf{u}, \boldsymbol{\lambda})=B\left(\boldsymbol{\lambda}, \mathbf{z}^{v}\right) \quad \forall \boldsymbol{\lambda} \in Q .
$$


Due to the theorem of Lax-Milgram we know that for every control $\mathbf{u} \in Q$ and every right hand side $\mathbf{f} \in H^{-1}(\Omega)^{d}$ equation (2.2) possesses a weak solution. Hence, we define a continuous affine linear solution operator $\mathcal{S}: Q \rightarrow X$, by $\mathbf{y}=\mathcal{S} \mathbf{u}$. Furthermore, we introduce the reduced cost functional $j: Q \rightarrow \mathbb{R}$ by

$$
j(\mathbf{u}):=J(\mathcal{S} \mathbf{u}, \mathbf{u}) .
$$

Lemma 2.1. It holds

$$
j^{\prime}(\mathbf{u})(\mathbf{q})=-B\left(\mathbf{q}, \mathbf{z}^{v}\right)+\alpha(\mathbf{u}, \mathbf{q}) \quad \forall \mathbf{u}, \mathbf{q} \in Q,
$$

where $\mathbf{z}=\left(\mathbf{z}^{v}, z^{p}\right) \in X$ is the solution of the adjoint equation (2.3).

Proof. This identity follows directly by applying the chain rule.

\section{Discretization of the optimality System}

\subsection{Finite element spaces}

In order to discretize the optimality system (2.2)-(2.4) we use conforming equal order finite elements. Let $\left\{\mathcal{T}_{h}\right\}$ be a family of shape-regular, admissible decompositions of $\Omega$ into $d$-dimensional quadrilaterals $(d=2)$ or hexahedra $(d=3)$. By $h_{K}$ we denote the diameter of a cell $K \in \mathcal{T}_{h}$. The maximal mesh size is $h=\max _{K \in \mathcal{T}_{h}} h_{K}$. We assume that for each $K \in \mathcal{T}_{h}$ there exists a bilinear mapping $F_{K}: \hat{K} \rightarrow K$ which maps the reference element $\hat{K}$ onto $K$. Let $Q_{r}$ be the space of all polynomials on the reference cell $\hat{K}$ with maximal degree $r \geq 1$ in each coordinate direction.

Now, we set $Q_{h}^{r}:=\left\{\varphi \in L^{2}(\Omega): \varphi \circ F_{K} \in Q_{r}, K \in \mathcal{T}_{h}\right\}$. The discrete state, dual and control spaces are given in the following way for $r, l \geq 1, m \geq 0$ :

$$
\begin{aligned}
X_{h} & :=X \cap\left[Q_{h}^{r} \cap C(\bar{\Omega})\right]^{d+1}, \\
Z_{h} & :=X \cap\left[Q_{h}^{l} \cap C(\bar{\Omega})\right]^{d+1}, \\
Q_{h} & :=Q \cap\left[Q_{h}^{m} \cap C(\bar{\Omega})\right]^{d} .
\end{aligned}
$$

By $I_{h}: C(\bar{\Omega}) \rightarrow Q_{h}^{r} \cap C(\bar{\Omega})$ we denote the Lagrange interpolation and use the bold notation $\boldsymbol{I}_{h}$ for the vector valued variant. In this work, the expression $a \lesssim b$ stands for $a \leq c b$ with a constant $c$ independent of the parameters $\alpha, \sigma, \mu$ and independent of $h$.

\subsection{Discrete variational formulation}

Our choice of finite element spaces consists of equal order interpolation for $p$ and $\mathbf{v}$. Hence, the discrete inf-sup condition is not fulfilled. Furthermore, in the convection dominated case there may occur unphysical oscillations. Hence, we need to add a stabilization term $S_{h}$ to the Galerkin formulation in order to cure these two types of instabilities. Different choices of this term and their effect to the discrete optimality system will be shown later on.

The equation for the discrete state $\mathbf{y}_{h} \in X_{h}$ reads now

$$
A\left(\mathbf{y}_{h}, \boldsymbol{\varphi}\right)+B\left(\mathbf{u}_{h}, \phi\right)+S_{h}\left(\mathbf{y}_{h}, \mathbf{u}_{h}\right)(\boldsymbol{\varphi})=\left\langle\mathbf{f}_{h}, \boldsymbol{\varphi}\right\rangle \quad \forall \boldsymbol{\varphi}=(\boldsymbol{\phi}, \xi) \in X_{h} .
$$

Furthermore, the right-hand side is changed to $\mathbf{f}_{h}$ which also may involve stabilization terms. If the discrete state equation (3.1) is uniquely solvable, we can define the discrete solution operator analogously to the continuous case by $\mathcal{S}_{h}: Q \rightarrow X_{h}, \mathcal{S}_{h} \mathbf{u}=\mathbf{y}_{h}(\mathbf{u})$. Furthermore, the discrete reduced functional is given by $j_{h}(\mathbf{u}):=J\left(\mathcal{S}_{h} \mathbf{u}, \mathbf{u}\right)$. 
Lemma 3.1. If (3.1) has an unique solution, then the second derivative of the discrete reduced cost functional $j_{h}$ is coercive:

$$
j_{h}^{\prime \prime}(\mathbf{u}, \mathbf{u})=\alpha\|\mathbf{u}\|_{0}^{2}+\left\|\mathcal{S}_{h} \mathbf{u}\right\|_{0}^{2} \quad \forall \mathbf{u} \in Q .
$$

Proof. Follows directly by definition of the functional $j_{h}$.

The adjoint equation (2.3) is also of Oseen type and therefore it also requires stabilization. We denote the corresponding stabilization term by $S_{h}^{z}$ and give the specific realization later. $S_{h}^{z}$ should be appropriate for the strong formulation (2.3).

Many stabilization methods for the Oseen problem are additive in the sense that it holds

$$
S_{h}\left(\mathbf{y}_{h}, \mathbf{u}_{h}\right)(\boldsymbol{\varphi})=S_{h}\left(\mathbf{y}_{h}, 0\right)(\varphi)+S_{h}\left(0, \mathbf{u}_{h}\right)(\boldsymbol{\varphi}) .
$$

In the following subsections, we give two examples of such stabilizations leading to unique solvability of (3.1). For the further analysis it is useful to introduce the discrete bilinear forms

$$
\begin{aligned}
& A_{h}(\mathbf{y}, \boldsymbol{\varphi}):=A(\mathbf{y}, \boldsymbol{\varphi})+S_{h}(\mathbf{y}, \mathbf{0})(\boldsymbol{\varphi}), \\
& B_{h}(\mathbf{u}, \boldsymbol{\varphi}):=B(\mathbf{u}, \phi)+S_{h}(\mathbf{0}, \mathbf{u})(\boldsymbol{\varphi}) .
\end{aligned}
$$

The optimality system for the discretize-optimize strategy becomes:

$$
\begin{array}{lll}
\mathbf{y}_{h} \in X_{h}: & A_{h}\left(\mathbf{y}_{h}, \boldsymbol{\varphi}\right)+B_{h}\left(\mathbf{u}_{h}, \boldsymbol{\varphi}\right)=\left\langle\mathbf{f}_{h}, \boldsymbol{\varphi}\right\rangle & \forall \boldsymbol{\varphi}=(\boldsymbol{\phi}, \xi) \in X_{h}, \\
\mathbf{z}_{h} \in X_{h}: & A_{h}\left(\boldsymbol{\psi}, \mathbf{z}_{h}\right)-\left(\mathbf{v}_{h}, \boldsymbol{\psi}^{v}\right)=-\left(\mathbf{v}_{d}, \boldsymbol{\psi}^{v}\right) & \forall \boldsymbol{\psi}=\left(\boldsymbol{\psi}^{v}, \psi^{p}\right) \in X_{h}, \\
\mathbf{u}_{h} \in Q_{h}: & B_{h}\left(\boldsymbol{\lambda}, \mathbf{z}_{h}\right)-\alpha\left(\mathbf{u}_{h}, \boldsymbol{\lambda}\right)=0 & \forall \boldsymbol{\lambda} \in Q_{h} .
\end{array}
$$

The optimality system for the optimize-discretize- strategy becomes:

$$
\begin{array}{llll}
\mathbf{y}_{h} \in X_{h}: & A_{h}\left(\mathbf{y}_{h}, \boldsymbol{\varphi}\right)+B_{h}\left(\mathbf{u}_{h}, \boldsymbol{\varphi}\right) & =\left\langle\mathbf{f}_{h}, \boldsymbol{\varphi}\right\rangle & \forall \boldsymbol{\varphi}=(\boldsymbol{\phi}, \xi) \in X_{h}, \\
\mathbf{z}_{h} \in X_{h}: & A\left(\boldsymbol{\psi}, \mathbf{z}_{h}\right)+S_{h}^{z}\left(\mathbf{z}_{h}, \mathbf{v}_{d}-\mathbf{v}_{h}\right)(\boldsymbol{\psi})-\left(\mathbf{v}_{h}, \boldsymbol{\psi}^{v}\right)=-\left(\mathbf{v}_{d}, \boldsymbol{\psi}^{v}\right) & \forall \boldsymbol{\psi}=\left(\boldsymbol{\psi}^{v}, \psi^{p}\right) \in X_{h}, \\
\mathbf{u}_{h} \in Q_{h}: & B\left(\boldsymbol{\lambda}, \mathbf{z}_{h}^{v}\right)-\alpha\left(\mathbf{u}_{h}, \boldsymbol{\lambda}\right) & =0 & \forall \boldsymbol{\lambda} \in Q_{h} .
\end{array}
$$

Note that the discrete primal equations (3.3) and (3.6) are identical, independent of the type of stabilization scheme. When the variables are ordered as $\left(\mathbf{u}_{h}, \mathbf{z}_{h}, \mathbf{y}_{h}\right)$, the linear operator corresponding to the system (3.3)-(3.5) turns out to be

$$
\left(\begin{array}{rrr}
-\alpha \mid & 0 & \mathrm{~B}_{h}^{*} \\
0 & -\mid & \mathrm{A}_{h}^{*} \\
\mathrm{~B}_{h} & \mathrm{~A}_{h} & 0
\end{array}\right),
$$

with appropriate matrices $\mathrm{A}_{h}, \mathrm{~B}_{h}$. Hence, this system is symmetric when $A_{h}$ is symmetric.

\subsection{SUPG/PSPG-stabilization}

For the classical combination of pressure stabilized Petrov-Galerkin (PSPG) and streamline upwind PetrovGalerkin (SUPG) [7] the stabilization form $S_{h}=S_{h}^{s d}$ reads

$$
S_{h}^{s d}\left(\mathbf{y}_{h}, \mathbf{u}_{h}\right)(\boldsymbol{\varphi}):=\sum_{K \in \mathcal{T}_{h}}\left\{\delta_{K}\left(-\mu \Delta \mathbf{v}_{h}+(\mathbf{b} \cdot \nabla) \mathbf{v}_{h}+\sigma \mathbf{v}_{h}+\nabla p_{h}+\mathbf{u}_{h},(\mathbf{b} \cdot \nabla) \boldsymbol{\phi}+\nabla \xi\right)_{K}+\gamma_{K}\left(\nabla \cdot \mathbf{v}_{h}, \nabla \cdot \boldsymbol{\phi}\right)_{K}\right\} .
$$

The new parameters $\delta_{K}$ and $\gamma_{K}$ are cell-wise constants. The corresponding right hand side becomes

$$
\left\langle\mathbf{f}_{h}, \varphi\right\rangle:=\langle\mathbf{f}, \phi\rangle+\sum_{K \in \mathcal{T}_{h}} \delta_{K}(\mathbf{f},(\mathbf{b} \cdot \nabla) \phi+\nabla \xi)_{K} .
$$


Obviously, this method allows the splitting (3.2). Related to this type of stabilization is the following norm:

$$
\|\mathbf{y}\|_{s d}:=\left(\mu|\mathbf{v}|_{1}^{2}+\sigma\|\mathbf{v}\|_{0}^{2}+\rho\|p\|_{0}^{2}+\sum_{K \in \mathcal{T}_{h}} \delta_{K}\|(\mathbf{b} \cdot \nabla) \mathbf{v}+\nabla p\|_{0, K}^{2}+\gamma_{K}\|\nabla \cdot \mathbf{v}\|_{0, K}^{2}\right)^{1 / 2},
$$

with sufficient small parameter $\rho>0$, for details see [14]. This definition of the norm provides additional control of the $L^{2}$-norm of the pressure, but the bilinear form $A_{h}(\cdot, \cdot)$ does not fulfill the coercivity estimate. However, with the following definitions of the parameters

$$
\gamma_{K}=\gamma_{0} h_{K}, \quad \delta_{K}=\delta_{0} \frac{h_{K}^{2}}{\|\mathbf{b}\|_{0, \infty ; K} h_{K}+\sigma h_{K}^{2}+\mu},
$$

with fixed $\delta_{0}, \gamma_{0}>0$, the bilinear form satisfies the discrete inf-sup condition

$$
\exists \beta>0, \text { s.t. } \quad \inf _{\mathbf{y}_{h} \in X_{h}} \sup _{\varphi \in X_{h}} \frac{A_{h}\left(\mathbf{y}_{h}, \boldsymbol{\varphi}\right)}{\left\|\mathbf{y}_{h}\right\| s d\|\boldsymbol{\varphi}\|_{s d}} \geq \beta,
$$

which guarantees existence and uniqueness of the discrete solution, see e.g. [15] for arbitrary polynomial order. We assume in the subsequent analysis that the stabilization parameters are chosen according to (3.9).

Corollary 3.2. The adjoint bilinear form (3.4) also fulfills the discrete inf-sup condition with the same choice of parameters (3.9) and constant $\beta>0$ from (3.10), e.g.

$$
\inf _{\mathbf{z}_{h} \in X_{h}} \sup _{\mathbf{u}_{h} \in X_{h}} \frac{A_{h}\left(\mathbf{u}_{h}, \mathbf{z}_{h}\right)}{\left\|\mathbf{u}_{h}\right\|_{s d}\left\|\mathbf{z}_{h}\right\|_{s d}} \geq \beta
$$

Proof. Let $\mathcal{A}_{h}: X_{h} \rightarrow X_{h}^{*}$ denote the linear operator associated with the bilinear form $A_{h}(\cdot, \cdot)$ by

$$
\mathbf{y}_{h} \mapsto A_{h}\left(\mathbf{y}_{h}, \cdot\right) .
$$

Due to the inf-sup condition (3.10) the operator $\mathcal{A}_{h}$ is injective. Furthermore, due to the fact that we consider a map between spaces of the same finite dimension, the mapping is also surjective. It follows for arbitrary $\mathbf{z}_{h} \in X_{h}$

$$
\left\|\mathbf{z}_{h}\right\|_{s d}=\sup _{\mathbf{g} \in X_{h}^{*}} \frac{\left\langle\mathbf{g}, \mathbf{z}_{h}\right\rangle}{\|\mathbf{g}\|_{X_{h}^{*}}}=\sup _{\mathbf{u}_{h} \in X_{h}} \frac{\left\langle\mathcal{A}_{h} \mathbf{u}_{h}, \mathbf{z}_{h}\right\rangle}{\left\|\mathcal{A}_{h} \mathbf{u}_{h}\right\|_{X_{h}^{*}}}=\sup _{\mathbf{u}_{h} \in X_{h}} \frac{A_{h}\left(\mathbf{u}_{h}, \mathbf{z}_{h}\right)}{\left\|\mathcal{A}_{h} \mathbf{u}_{h}\right\|_{X_{h}^{*}}} \leq \sup _{\mathbf{u}_{h} \in X_{h}} \frac{A_{h}\left(\mathbf{u}_{h}, \mathbf{z}_{h}\right)}{\beta\left\|\mathbf{u}_{h}\right\|_{s d}} .
$$

\subsection{Local projection stabilization}

The main idea of local projection stabilization (LPS) is to include artificial viscosity but only for the fine scale part of the pressure gradient and of the velocity gradient in the stabilization term. For simplicity, we restrict here to the so called two-level version of LPS. However, all results stated in the following are also valid for one-level variants [17].

Let $\mathcal{T}_{2 h}$ be the coarser mesh obtained by a "global coarsening" of $\mathcal{T}_{h}$. The finer mesh $\mathcal{T}_{h}$ contains $2^{d}$ times more elements than $\mathcal{T}_{2 h}$. The elements of $\mathcal{T}_{2 h}$ will be denoted by "patches". By $\pi_{h}$ we denote the $L^{2}$-projection operator on discontinuous finite elements

$$
\pi_{h}: L^{2}(\Omega) \rightarrow Q_{2 h}^{r-1},
$$

characterized by the property for $v \in L^{2}(\Omega)$ :

$$
\left(v-\pi_{h} v, \phi\right)=0 \quad \forall \phi \in Q_{2 h}^{r-1} .
$$


Important is the fact that this projection acts locally on patches of elements, so that the numerical effort for computing this projection is very low. The operator giving the space fluctuations is denoted by

$$
\varkappa_{h}:=I-\pi_{h} .
$$

with the identity mapping $I$. We use the bold notations $\boldsymbol{\pi}_{h}, \boldsymbol{\varkappa}_{h}$ for the mappings of vector-valued functions, for instance, $\boldsymbol{\pi}_{h}: L^{2}(\Omega)^{d} \rightarrow\left[Q_{2 h}^{r-1}\right]^{d}$.

The discrete primal equation of the optimal control problem with local projection is as in (3.1) with a stabilization term independent of the control $\mathbf{u}$ :

$$
\begin{aligned}
& \left.S_{h}^{l p s}(\mathbf{y}, 0)(\boldsymbol{\varphi}):=\left((\mathbf{b} \cdot \nabla) \mathbf{v}, \delta \varkappa_{h}[(\mathbf{b} \cdot \nabla) \phi)\right]\right)+\left(\nabla p, \tau \varkappa_{h} \nabla \xi\right)+\left(\nabla \cdot \mathbf{v}, \gamma \varkappa_{h}(\nabla \cdot \boldsymbol{\phi})\right), \\
& S_{h}^{l p s}(\mathbf{0}, \mathbf{u})(\boldsymbol{\varphi}):=0 .
\end{aligned}
$$

The parameters $\tau, \delta$ and $\gamma$ are patch-wise constant and depend (similar to PSPG and SUPG) on the local Peclet number. This bilinear stabilization is proposed in [2] and analyzed in [5]. Due to the orthogonality property (3.12), it is easy to verify, that $S_{h}^{l p s}(\mathbf{y}, \mathbf{u})(\boldsymbol{\varphi})$ is symmetric, i.e. $S_{h}^{l p s}(\mathbf{y}, \mathbf{u})(\boldsymbol{\varphi})=S_{h}^{l p s}(\boldsymbol{\varphi}, \mathbf{u})(\mathbf{y})$.

Associated to this method is the semi-norm

$$
\|\mathbf{y}\|_{l p s}:=\left(\mu|\mathbf{v}|_{1}^{2}+\sigma\|\mathbf{v}\|_{0}^{2}+S_{h}^{l p s}(\mathbf{y}, 0)(\mathbf{y})\right)^{1 / 2} .
$$

Note that this semi-norm does not include the $L^{2}$-norm of $p$. However, an a priori estimate of $\left\|p-p_{h}\right\|$ with optimal order can be easily achieved as shown in [5].

\section{A PRIORI ERROR ANALYSIS}

In this section we present an a priori error estimate of the linear-quadratic optimal control problem (1.1)-(1.2) with stabilized finite elements of the above types. The estimates for the PSPG/SUPG method are new. The estimate for LPS fits into the framework in [4]. However, in order to have a comparison, we state also the result for LPS.

For an upper bound of interpolation errors in the norm $\|\cdot\|_{s d}$ we will use the following notation for $\mathbf{y}=(\mathbf{v}, p)$ :

$$
\varepsilon(\mathbf{y}):=\left(\sum_{K \in \mathcal{T}_{h}}\left(L_{K} h_{K}^{2 r}\|\mathbf{v}\|_{r+1, K}^{2}+h_{K}^{2 r+1}\|p\|_{r+1, K}^{2}\right)\right)^{1 / 2},
$$

with $L_{K}:=\mu+\sigma h_{K}^{2}+h_{K}\|\mathbf{b}\|_{\infty, K}+h_{K}$. In order to describe the $\sigma$ and $\mu$ dependence of the error we will use the notation

$$
\eta:=\left(\sigma+\frac{\mu}{c_{\Omega}}\right)^{-1}
$$

where $c_{\Omega}$ is the Poincaré constant in $\|\phi\|_{0} \leq c_{\Omega}|\phi|_{1}$ for $\phi \in H_{0}^{1}(\Omega)$.

Lemma 4.1. Let $\mathbf{y}$ the solution of (2.2). If $\mathbf{y} \in H^{r+1}(\Omega)^{d+1}$, then we obtain for the Lagrange interpolation $I_{h}$ and the parameter choice (3.9):

$$
\left\|\mathbf{y}-\boldsymbol{I}_{h} \mathbf{y}\right\|_{l p s}+\left\|\mathbf{y}-\boldsymbol{I}_{h} \mathbf{y}\right\|_{s d} \lesssim \varepsilon(\mathbf{y}) .
$$

Proof. This is a direct implication of the standard estimate for $k \in\{0,1\}$ :

$$
\left\|\phi-I_{h} \phi\right\|_{k}^{2} \lesssim \sum_{K \in \mathcal{T}_{h}} h_{K}^{2(r+1-k)}\|\phi\|_{r+1, K}^{2} \quad \forall \phi \in H^{r+1}(\Omega)
$$

and definition (3.9). 


\subsection{First discretize then optimize for PSPG/SUPG}

Lemma 4.2. Let $\mathbf{v}_{h}$ be the velocity component of $\mathcal{S}_{h} \mathbf{u}$ and $\mathbf{z}_{h} \in X_{h}$ the corresponding solution of the adjoint equation (3.4). Then there holds for all $\mathbf{u}, \boldsymbol{q} \in Q$

$$
j_{h}^{\prime}(\mathbf{u})(\boldsymbol{q})=-B_{h}\left(\boldsymbol{q}, \mathbf{z}_{h}\right)+\alpha(\mathbf{u}, \boldsymbol{q}) .
$$

Proof. The derivative of the discrete reduced functional can be expressed by

$$
\begin{aligned}
j_{h}^{\prime}(\mathbf{u})(\boldsymbol{q}) & =\partial_{u} J\left(\mathcal{S}_{h} \mathbf{u}, \mathbf{u}\right)(\boldsymbol{q}) \\
& =\left(\mathcal{S}_{h}^{\prime}(\mathbf{u})(\boldsymbol{q}), \mathbf{v}_{h}-\mathbf{v}_{d}\right)+\alpha(\mathbf{u}, \boldsymbol{q}) .
\end{aligned}
$$

Since $\mathcal{S}$ is affine-linear, it holds $\mathcal{S}_{h}^{\prime}(\mathbf{u})(\boldsymbol{q})=\mathcal{S}_{h}(\mathbf{u}+\boldsymbol{q})-\mathcal{S}_{h} \mathbf{u}$. With $\mathbf{z}_{h} \in X_{h}$, the solution of the adjoint equation (3.4), we obtain

$$
\left(\mathcal{S}_{h}^{\prime}(\mathbf{u})(\boldsymbol{q}), \mathbf{v}_{h}-\mathbf{v}_{d}\right)=A_{h}\left(\mathcal{S}_{h}(\mathbf{u}+\boldsymbol{q}), \mathbf{z}_{h}\right)-A_{h}\left(\mathcal{S}_{h} \mathbf{u}, \mathbf{z}_{h}\right)=-B_{h}\left(\boldsymbol{q}, \mathbf{z}_{h}\right) .
$$

This implies the assertion.

Lemma 4.3. For given controls $\mathbf{u}, \widehat{\mathbf{u}} \in Q$ let $\mathbf{y}(\mathbf{u}) \in X$ be the solution of the state equation associated to $\mathbf{u}$ and $\widehat{\mathbf{y}}_{h}(\hat{\mathbf{u}}) \in X_{h}$ be the discrete solution associated to $\widehat{\mathbf{u}}$. Then the following error estimate holds:

$$
\left\|\mathbf{y}-\widehat{\mathbf{y}}_{h}\right\|_{s d} \lesssim \eta^{1 / 2}\|\mathbf{u}-\widehat{\mathbf{u}}\|_{0}+\varepsilon(\mathbf{y})
$$

Proof. The inf-sup condition (3.10) gives us

$$
\left\|\boldsymbol{I}_{h} \mathbf{y}-\widehat{\mathbf{y}}_{h}\right\|_{s d} \leq \frac{1}{\beta} \sup _{\boldsymbol{\varphi} \in X_{h}} \frac{A_{h}\left(\boldsymbol{I}_{h} \mathbf{y}-\widehat{\mathbf{y}}_{h}, \boldsymbol{\varphi}\right)}{\|\boldsymbol{\varphi}\|_{s d}} .
$$

Due to Galerkin orthogonality we estimate

$$
\begin{aligned}
A_{h}\left(\boldsymbol{I}_{h} \mathbf{y}-\widehat{\mathbf{y}}_{h}, \boldsymbol{\varphi}\right) & =A_{h}\left(\boldsymbol{I}_{h} \mathbf{y}-\mathbf{y}, \boldsymbol{\varphi}\right)-B_{h}(\mathbf{u}-\widehat{\mathbf{u}}, \boldsymbol{\phi}) \\
& \leq A_{h}\left(\boldsymbol{I}_{h} \mathbf{y}-\mathbf{y}, \boldsymbol{\varphi}\right)+\left(\eta^{1 / 2}+\left(\max _{K} \delta_{K}\right)^{1 / 2}\right)\|\mathbf{u}-\widehat{\mathbf{u}}\|_{0}\|\boldsymbol{\varphi}\|_{s d} .
\end{aligned}
$$

Furthermore we estimate

$$
A_{h}\left(\boldsymbol{I}_{h} \mathbf{y}-\mathbf{y}, \boldsymbol{\varphi}\right)=A\left(\boldsymbol{I}_{h} \mathbf{y}-\mathbf{y}, \boldsymbol{\varphi}\right)+S_{h}^{s d}\left(\boldsymbol{I}_{h} \mathbf{y}-\mathbf{y}, \mathbf{0}\right)(\boldsymbol{\varphi}) .
$$

The Galerkin part is bounded as follows:

$$
\begin{aligned}
A\left(\boldsymbol{I}_{h} \mathbf{y}-\mathbf{y}, \boldsymbol{\varphi}\right)= & \mu\left(\nabla\left(\boldsymbol{I}_{h} \mathbf{v}-\mathbf{v}\right), \nabla \boldsymbol{\phi}\right)+\sigma\left(\boldsymbol{I}_{h} \mathbf{v}-\mathbf{v}, \boldsymbol{\phi}\right)-\left(\boldsymbol{I}_{h} \mathbf{v}-\mathbf{v},(\mathbf{b} \cdot \nabla) \boldsymbol{\phi}+\nabla \xi\right)-\left(I_{h} p-p, \nabla \cdot \boldsymbol{\phi}\right) \\
\leq & 2\left\|\boldsymbol{I}_{h} \mathbf{y}-\mathbf{y}\right\|_{s d}\|\boldsymbol{\varphi}\|_{s d}+\sum_{K \in \mathcal{T}_{h}} \delta_{K}^{-1 / 2}\left\|\boldsymbol{I}_{h} \mathbf{v}-\mathbf{v}\right\|_{0 ; K} \delta_{K}^{1 / 2}\|(\mathbf{b} \cdot \nabla) \boldsymbol{\phi}+\nabla \xi\|_{0 ; K} \\
& +\sum_{K \in \mathcal{T}_{h}} \gamma_{K}^{-1 / 2}\left\|I_{h} p-p\right\|_{0 ; K} \gamma_{K}^{1 / 2}\|\nabla \cdot \boldsymbol{\phi}\|_{0 ; K} \\
\lesssim & \|\boldsymbol{\varphi}\|_{s d}\left[\left\|\boldsymbol{I}_{h} \mathbf{y}-\mathbf{y}\right\|_{s d}+\left(\sum_{K \in \mathcal{T}_{h}} \delta_{K}^{-1}\left\|\boldsymbol{I}_{h} \mathbf{v}-\mathbf{v}\right\|_{0 ; K}^{2}\right)^{1 / 2}+\left(\sum_{K \in \mathcal{T}_{h}} \gamma_{K}^{-1}\left\|I_{h} p-p\right\|_{0 ; K}^{2}\right)^{1 / 2}\right] \\
\lesssim & \|\boldsymbol{\varphi}\|_{s d} \varepsilon(\mathbf{y}) .
\end{aligned}
$$


For the stabilization term we obtain due to $\|\nabla \cdot \mathbf{v}\|_{0} \leq \sqrt{d}\|\nabla \mathbf{v}\|_{0}$

$$
\begin{aligned}
S_{h}^{s d}\left(\boldsymbol{I}_{h} \mathbf{y}-\mathbf{y}, \mathbf{0}\right)(\boldsymbol{\varphi})= & \sum_{K \in \mathcal{T}_{h}}\left\{\delta_{K}\left(-\mu \Delta\left(\boldsymbol{I}_{h} \mathbf{v}-\mathbf{v}\right)+(\mathbf{b} \cdot \nabla)\left(\boldsymbol{I}_{h} \mathbf{v}-\mathbf{v}\right)+\sigma\left(\boldsymbol{I}_{h} \mathbf{v}-\mathbf{v}\right)+\nabla\left(I_{h} p-p\right),(\mathbf{b} \cdot \nabla) \boldsymbol{\phi}+\nabla \xi\right)_{K}\right. \\
& \left.+\gamma_{K}\left(\nabla \cdot\left(\boldsymbol{I}_{h} \mathbf{v}-\mathbf{v}\right), \nabla \cdot \boldsymbol{\phi}\right)_{K}\right\} \\
\leq & \|\boldsymbol{\varphi}\|_{s d}\left(\sum _ { K \in \mathcal { T } _ { h } } \delta _ { K } \left[\mu^{2}\left\|\Delta\left(\boldsymbol{I}_{h} \mathbf{v}-\mathbf{v}\right)\right\|_{0 ; K}^{2}+\|\mathbf{b}\|_{0, \infty ; K}^{2}\left\|\nabla\left(\boldsymbol{I}_{h} \mathbf{v}-\mathbf{v}\right)\right\|_{0 ; K}^{2}+\sigma^{2}\left\|\boldsymbol{I}_{h} \mathbf{v}-\mathbf{v}\right\|_{0 ; K}^{2}\right.\right. \\
& \left.\left.+\left\|\nabla\left(I_{h} p-p\right)\right\|_{0 ; K}^{2}\right]+\gamma_{K}\left\|\nabla\left(\boldsymbol{I}_{h} \mathbf{v}-\mathbf{v}\right)\right\|_{0 ; K}^{2}\right)^{1 / 2} .
\end{aligned}
$$

The assertion follows by the interpolation estimate of Lemma 4.1, definition (3.9) and via triangle inequality.

Lemma 4.4. For given controls $\mathbf{u}, \hat{\mathbf{u}} \in Q$ let $\mathbf{z}(\mathbf{y}(\mathbf{u})) \in X$ and $\hat{\mathbf{z}}_{h}\left(\hat{\mathbf{y}}_{h}(\hat{\mathbf{u}})\right) \in Z_{h}$ be the associated adjoint solutions to $\mathbf{u}$ and $\hat{\mathbf{u}}$, respectively. Then it holds:

$$
\left\|\mathbf{z}-\hat{\mathbf{z}}_{h}\right\|_{s d} \lesssim \varepsilon(\mathbf{z})+\eta^{3 / 2}\|\mathbf{u}-\widehat{\mathbf{u}}\|_{0}+(1+\eta) \varepsilon(\mathbf{y})+\sup _{\boldsymbol{\varphi} \in X_{h}} \frac{S_{h}^{s d}(\boldsymbol{\varphi}, \mathbf{0})\left(\boldsymbol{I}_{h} \mathbf{z}\right)}{\|\boldsymbol{\varphi}\|_{s d}}
$$

Proof. By $\hat{\mathbf{y}}_{h}$ we denote the discrete primal solution of (3.3) for the control $\hat{\mathbf{u}}$. For discretize-optimize with SUPG/PSPG the adjoint system is not stabilized in a consistent way. Therefore we obtain a perturbed Galerkin orthogonality by subtracting (3.4) from (2.3)

$$
A\left(\boldsymbol{\varphi}, \mathbf{z}-\hat{\mathbf{z}}_{h}\right)=S_{h}^{s d}(\boldsymbol{\varphi}, \mathbf{0})\left(\hat{\mathbf{z}}_{h}\right)+\left(\mathbf{v}-\hat{\mathbf{v}}_{h}, \phi\right) \quad \forall \boldsymbol{\varphi}=(\phi, \xi) \in X_{h} .
$$

With definitions $\boldsymbol{\eta}:=\mathbf{z}-\boldsymbol{I}_{h} \mathbf{z}$ and $\boldsymbol{\omega}:=\boldsymbol{I}_{h} \mathbf{z}-\hat{\mathbf{z}}_{h}$ and by using the triangle inequality we can split the error

$$
\left\|\mathbf{z}-\hat{\mathbf{z}}_{h}\right\|_{s d} \leq \varepsilon(\mathbf{z})+\|\boldsymbol{\omega}\|_{s d} .
$$

The second term can be estimated by help of (3.4):

$$
\begin{aligned}
\|\boldsymbol{\omega}\|_{s d} & \leq \frac{1}{\beta} \sup _{\boldsymbol{\varphi} \in X_{h}} \frac{A(\boldsymbol{\varphi}, \boldsymbol{\omega})+S_{h}^{s d}(\boldsymbol{\varphi}, \mathbf{0})(\boldsymbol{\omega})}{\|\boldsymbol{\varphi}\|_{s d}} \\
& =\frac{1}{\beta} \sup _{\boldsymbol{\varphi} \in X_{h}} \frac{A\left(\boldsymbol{\varphi}, \mathbf{z}-\hat{\mathbf{z}}_{h}\right)-A(\boldsymbol{\varphi}, \boldsymbol{\eta})-S_{h}^{s d}(\boldsymbol{\varphi}, \mathbf{0})\left(\hat{\mathbf{z}}_{h}\right)+S_{h}^{s d}(\boldsymbol{\varphi}, \mathbf{0})\left(\boldsymbol{I}_{h} \mathbf{z}\right)}{\|\boldsymbol{\varphi}\| s d} \\
& =\frac{1}{\beta} \sup _{\boldsymbol{\varphi} \in X_{h}} \frac{\left(\mathbf{v}-\hat{\mathbf{v}}_{h}, \boldsymbol{\phi}\right)-A(\boldsymbol{\varphi}, \boldsymbol{\eta})+S_{h}^{s d}(\boldsymbol{\varphi}, \mathbf{0})\left(\boldsymbol{I}_{h} \mathbf{z}\right)}{\|\boldsymbol{\varphi}\|_{s d}} .
\end{aligned}
$$

For the first term we obtain with the Poincare inequality and Lemma 4.3

$$
\begin{aligned}
\left(\mathbf{v}-\hat{\mathbf{v}}_{h}, \boldsymbol{\phi}\right) & \leq\left\|\mathbf{v}-\hat{\mathbf{v}}_{h}\right\|_{0}\|\boldsymbol{\phi}\|_{0} \\
& \lesssim \eta\left\|\mathbf{y}-\hat{\mathbf{y}}_{h}\right\|_{s d}\|\boldsymbol{\varphi}\|_{s d} \\
& \lesssim \eta\left(\varepsilon(\mathbf{y})+\eta^{1 / 2}\|\mathbf{u}-\widehat{\mathbf{u}}\|_{0}\right)\|\boldsymbol{\varphi}\|_{s d} .
\end{aligned}
$$

Analogously to Lemma 4.3 we obtain by help of the interpolation estimate (4.1) for the bilinear form $A$

$$
\begin{aligned}
A(\boldsymbol{\varphi}, \boldsymbol{\eta}) & \leq\|\boldsymbol{\varphi}\|_{s d}\left[\varepsilon(\mathbf{y})+\left(\sum_{K \in \mathcal{T}_{h}} \delta_{K}^{-1}\left\|\boldsymbol{I}_{h} \mathbf{v}-\mathbf{v}\right\|_{0 ; K}^{2}\right)^{1 / 2}+\left(\sum_{K \in \mathcal{T}_{h}} \gamma_{K}^{-1}\left\|I_{h} p-p\right\|_{0 ; K}^{2}\right)^{1 / 2}\right] \\
& \lesssim\|\boldsymbol{\varphi}\|_{s d} \varepsilon(\mathbf{y}) .
\end{aligned}
$$


In the following Lemma we derive an upper bound for the SUPG/PSPG term arising on the right hand side of Lemma 4.4.

Lemma 4.5. We define the stabilization parameter as indicated in (3.9). Then it holds for arbitrary $\varphi \in X_{h}$ and arbitrary $\mathbf{z}=\left(\mathbf{z}^{v}, z^{p}\right) \in X$ with $\nabla \cdot \mathbf{z}^{v}=0$ :

$$
S_{h}^{s d}(\boldsymbol{\varphi}, \mathbf{0})\left(\boldsymbol{I}_{h} \mathbf{z}\right) \lesssim\|\boldsymbol{\varphi}\|_{s d}\left(\sum_{K \in \mathcal{T}_{h}}\left(D_{K}^{2}\left(\boldsymbol{I}_{h} \mathbf{z}\right)+h_{K}^{2 l+1}\left\|\mathbf{z}^{v}\right\|_{l+1, K}^{2}\right)\right)^{1 / 2},
$$

with the notation $D_{K}(\mathbf{z}):=\delta_{K}^{1 / 2}\left\|(\mathbf{b} \cdot \nabla) \mathbf{z}^{v}+\nabla z^{p}\right\|_{0, K}$.

Proof. The stabilization term is of the following form:

$$
S_{h}^{s d}(\boldsymbol{\varphi}, \mathbf{0})\left(\boldsymbol{I}_{h} \mathbf{z}\right)=\sum_{K \in \mathcal{T}_{h}}\left\{\delta_{K}\left(-\mu \Delta \boldsymbol{\phi}+(\mathbf{b} \cdot \nabla) \boldsymbol{\phi}+\sigma \boldsymbol{\phi}+\nabla \xi,(\mathbf{b} \cdot \nabla) \boldsymbol{I}_{h} \mathbf{z}^{v}+\nabla \boldsymbol{I}_{h} z^{p}\right)_{K}+\gamma_{K}\left(\nabla \cdot \boldsymbol{\phi}, \nabla \cdot \boldsymbol{I}_{h} \mathbf{z}^{v}\right)_{K}\right\} .
$$

Using the abbreviation $E_{K}(\phi):=h_{K}^{1 / 2}\|\nabla \cdot \phi\|_{0}$, we get by an inverse estimate

$$
\begin{aligned}
\sum_{K \in \mathcal{T}_{h}} \delta_{K}\left(-\mu \Delta \phi,(\mathbf{b} \cdot \nabla) \boldsymbol{I}_{h} \mathbf{z}^{v}+\nabla I_{h} z^{p}\right)_{K} & \leq \sum_{K \in \mathcal{T}_{h}} \mu\|\Delta \phi\|_{0, K} \delta_{K}^{1 / 2} D_{K}\left(\boldsymbol{I}_{h} \mathbf{z}\right) \\
& \lesssim\left(\sum_{K \in \mathcal{T}_{h}} \mu \delta_{K} h_{K}^{-2} D_{K}^{2}\left(\boldsymbol{I}_{h} \mathbf{z}\right)\right)^{1 / 2} \mu^{1 / 2}|\boldsymbol{\phi}|_{1} \\
\sum_{K \in \mathcal{T}_{h}} \delta_{K}\left((\mathbf{b} \cdot \nabla) \phi+\nabla \xi,(\mathbf{b} \cdot \nabla) \boldsymbol{I}_{h} \mathbf{z}^{v}+\nabla I_{h} z^{p}\right)_{K} & \leq\left(\sum_{K \in \mathcal{T}_{h}} D_{K}^{2}\left(\boldsymbol{I}_{h} \mathbf{z}\right)\right)^{1 / 2}\left(\sum_{K \in \mathcal{T}_{h}} D_{K}^{2}(\boldsymbol{\varphi})\right)^{1 / 2} \\
\sum_{K \in \mathcal{T}_{h}} \delta_{K}\left(\sigma \phi,(\mathbf{b} \cdot \nabla) \boldsymbol{I}_{h} \mathbf{z}^{v}+\nabla I_{h} z^{p}\right) & \leq\left(\sum_{K \in \mathcal{T}_{h}} \sigma \delta_{K} D_{K}^{2}\left(\boldsymbol{I}_{h} \mathbf{z}\right)\right)^{1 / 2} \sigma^{1 / 2}\|\boldsymbol{\varphi}\|_{0} \\
\sum_{K \in \mathcal{T}_{h}} \gamma_{K}\left(\nabla \cdot \phi, \nabla \cdot \boldsymbol{I}_{h} \mathbf{z}^{v}\right) & \leq\left(\sum_{K \in \mathcal{T}_{h}} E_{K}^{2}\left(\boldsymbol{I}_{h} \mathbf{z}^{v}\right)\right)^{1 / 2}\left(\sum_{K \in \mathcal{T}_{h}} E_{K}^{2}(\boldsymbol{\phi})\right)^{1 / 2} .
\end{aligned}
$$

Altogether we can estimate the stabilization term by

$$
S_{s d}^{h}(\boldsymbol{\varphi}, \mathbf{0})\left(\boldsymbol{I}_{h} \mathbf{z}\right) \lesssim\left(\sum_{K \in \mathcal{T}_{h}}\left(M_{K} D_{K}^{2}\left(\boldsymbol{I}_{h} \mathbf{z}\right)+E_{K}^{2}\left(\boldsymbol{I}_{h} \mathbf{z}^{v}\right)\right)\right)^{1 / 2}\|\boldsymbol{\varphi}\|_{s d},
$$

with $M_{K}=1+h_{K}^{-2} \mu \delta_{K}+\delta_{K} \sigma$. Due to the fact that $\delta_{K} \leq \delta_{0} \sigma^{-1}$ and $\delta_{K} \leq \delta_{0} h_{K}^{2} / \mu, M_{K}$ can be bounded by $1+2 \delta_{0}$, i.e. by a $h$-independent constant. Now, we use that the velocity components of $\mathbf{z}$ are supposed to be divergence free:

$$
E_{K}\left(\boldsymbol{I}_{h} \mathbf{z}^{v}\right)=E_{K}\left(\mathbf{z}^{v}-\boldsymbol{I}_{h} \mathbf{z}^{v}\right) \lesssim h_{K}^{l+1 / 2}\left\|\mathbf{z}^{v}\right\|_{l+1, K}
$$

and arrive at the target estimate

$$
S_{h}^{s d}(\boldsymbol{\varphi}, \mathbf{0})\left(\boldsymbol{I}_{h} \mathbf{z}\right) \lesssim\|\boldsymbol{\varphi}\|_{s d}\left(\sum_{K \in \mathcal{T}_{h}}\left(D_{K}^{2}\left(\boldsymbol{I}_{h} \mathbf{z}\right)+h_{K}^{2 l+1}\left\|\mathbf{z}^{v}\right\|_{l+1, K}^{2}\right)\right)^{1 / 2} .
$$


Remark 4.6. Lemma 4.3 shows that the error in the state variable measured in the triplenorm is bounded by the $L^{2}$-error in the control plus interpolation error. Therefore, improved errors in the control carries over to the error in the state. However, combining Lemmas 4.4 and 4.5 the error in the adjoint state measured in the triplenorm is only of order $\mathcal{O}\left(h^{1 / 2}\right)$ due to the term

$$
D_{K}\left(\boldsymbol{I}_{h} \mathbf{z}\right)=\delta_{K}^{1 / 2}\left\|(\mathbf{b} \cdot \nabla) \boldsymbol{I}_{h} \mathbf{z}^{v}+\nabla I_{h} z^{p}\right\|_{0, K} \lesssim \min \left(h_{K}^{1 / 2}, \sigma^{-1 / 2}, h_{K} / \mu\right) .
$$

In the numerical examples we will demonstrate that this estimate is sharp.

Theorem 4.7. Let $(\mathbf{y}, \mathbf{z}, \mathbf{u}) \in X \times X \times Q$ be the solution of the continuous optimization problem (2.2)-(2.4) and $\left(\mathbf{y}_{h}, \mathbf{z}_{h}, \mathbf{u}_{h}\right) \in X_{h} \times X_{h} \times Q_{h}$ be the discrete solution of (3.3)-(3.5) for SUPG/PSPG. Then it holds

$$
\left\|\mathbf{u}-\mathbf{u}_{h}\right\|_{0} \lesssim \lambda\left\|\mathbf{z}-\mathbf{z}_{h}\left(\boldsymbol{I}_{h} \mathbf{u}\right)\right\|_{s d}+\left\|\mathbf{u}-\boldsymbol{I}_{h} \mathbf{u}\right\|_{0}+\frac{1}{\alpha}\left(\sum_{K \in \mathcal{T}_{h}} D_{K}^{2}(\mathbf{z})\right)^{1 / 2},
$$

with $\lambda:=\frac{1}{\alpha}(1+\eta)^{1 / 2}$ and $D_{K}(\mathbf{z})$ as defined in the previous Lemma.

Proof. Obviously, it is sufficient to have an appropriate bound on $\left\|\boldsymbol{I}_{h} \mathbf{u}-\mathbf{u}_{h}\right\|$. Even for arbitrary $\hat{\mathbf{u}}_{h} \in Q_{h}$ it holds due to Lemma 3.1 and due to the fact that $j_{h}$ is quadratic

$$
\begin{aligned}
\alpha\left\|\hat{\mathbf{u}}_{h}-\mathbf{u}_{h}\right\|_{0}^{2} & \leq j_{h}^{\prime \prime}\left(\hat{\mathbf{u}}_{h}-\mathbf{u}_{h}, \hat{\mathbf{u}}_{h}-\mathbf{u}_{h}\right) \\
& =j_{h}^{\prime}\left(\hat{\mathbf{u}}_{h}\right)\left(\hat{\mathbf{u}}_{h}-\mathbf{u}_{h}\right)-j_{h}^{\prime}\left(\mathbf{u}_{h}\right)\left(\hat{\mathbf{u}}_{h}-\mathbf{u}_{h}\right) .
\end{aligned}
$$

Since $j_{h}^{\prime}\left(\mathbf{u}_{h}\right)\left(\hat{\mathbf{u}}_{h}-\mathbf{u}_{h}\right)=0=j^{\prime}(\mathbf{u})\left(\hat{\mathbf{u}}_{h}-\mathbf{u}_{h}\right)$ we get the upper bound

$$
\alpha\left\|\hat{\mathbf{u}}_{h}-\mathbf{u}_{h}\right\|_{0}^{2} \leq j_{h}^{\prime}\left(\hat{\mathbf{u}}_{h}\right)\left(\hat{\mathbf{u}}_{h}-\mathbf{u}_{h}\right)-j^{\prime}(\mathbf{u})\left(\hat{\mathbf{u}}_{h}-\mathbf{u}_{h}\right) .
$$

Using Lemmas 2.1 and 4.2 we obtain

$$
\begin{aligned}
\alpha\left\|\hat{\mathbf{u}}_{h}-\mathbf{u}_{h}\right\|_{0}^{2} \leq & -B\left(\hat{\mathbf{u}}_{h}-\mathbf{u}_{h}, \hat{\mathbf{z}}_{h}\right)+\alpha\left(\hat{\mathbf{u}}_{h}, \hat{\mathbf{u}}_{h}-\mathbf{u}_{h}\right)+S_{h}^{s d}\left(\mathbf{0}, \hat{\mathbf{u}}_{h}-\mathbf{u}_{h}\right)\left(\hat{\mathbf{z}}_{h}-\mathbf{z}+\mathbf{z}\right) \\
& +B\left(\hat{\mathbf{u}}_{h}-\mathbf{u}_{h}, \mathbf{z}\right)-\alpha\left(\mathbf{u}, \hat{\mathbf{u}}_{h}-\mathbf{u}_{h}\right) \\
\lesssim & \left\|\hat{\mathbf{u}}_{h}-\mathbf{u}_{h}\right\|_{0}\left(\left\|\hat{\mathbf{z}}_{h}-\mathbf{z}\right\|_{0}+\alpha\left\|\hat{\mathbf{u}}_{h}-\mathbf{u}\right\|_{0}+\left\|\hat{\mathbf{z}}_{h}-\mathbf{z}\right\|_{s d}+\left(\sum_{K \in \mathcal{T}_{h}} D_{K}^{2}(\mathbf{z})\right)^{1 / 2}\right) .
\end{aligned}
$$

Here, we used due to $\delta_{K} \leq C$ the upper bound

$$
\left|\sum_{K} \delta_{K}(\tilde{\mathbf{u}},(\mathbf{b} \cdot \nabla) \mathbf{v}+\nabla p)_{K}\right| \lesssim\|\tilde{\mathbf{u}}\|_{0}\|\mathbf{y}\|_{s d} \quad \forall \mathbf{y}=(\mathbf{v}, p) \in X, \forall \tilde{\mathbf{u}} \in Q .
$$

Therefore, we get

$$
\left\|\hat{\mathbf{u}}_{h}-\mathbf{u}_{h}\right\|_{0} \lesssim \frac{1}{\alpha}\left\|\hat{\mathbf{z}}_{h}-\mathbf{z}\right\|_{0}+\frac{1}{\alpha}\left\|\mathbf{z}-\hat{\mathbf{z}}_{h}\right\|_{s d}+\left\|\hat{\mathbf{u}}_{h}-\mathbf{u}\right\|_{0}+\frac{1}{\alpha}\left(\sum_{K \in \mathcal{T}_{h}} D_{K}^{2}(\mathbf{z})\right)^{1 / 2} .
$$

Now, we choose $\hat{\mathbf{u}}_{h}=\boldsymbol{I}_{h} u$ and apply the triangle inequality in order to obtain the desired estimate.

The following Corollary shows that the order of convergence in the control is at least $\mathcal{O}\left(h^{1 / 2}\right)$. 
Corollary 4.8. Let the assumptions from Theorem 4.7 be fulfilled and assume the following regularity assumptions on the continuous solutions for integers $r, l \geq 1$ and $m \geq 0$ :

$$
\mathbf{y} \in H^{r+1}(\Omega)^{d+1}, \quad \mathbf{z} \in H^{l+1}(\Omega)^{d+1}, \quad \mathbf{u} \in H^{m+1}(\Omega)^{d} .
$$

Then it holds with $\lambda:=\frac{1}{\alpha}(1+\eta)^{1 / 2}$,

$$
\left\|\mathbf{u}-\mathbf{u}_{h}\right\|_{0} \lesssim \lambda \varepsilon(\mathbf{z})+\lambda(1+\eta) \varepsilon(\mathbf{y})+\left(1+\lambda \eta^{3 / 2}\right)\left\|\mathbf{u}-\boldsymbol{I}_{h} \mathbf{u}\right\|+\left(\lambda+\alpha^{-1}\right)\left(\sum_{K \in \mathcal{T}_{h}} D_{K}^{2}(\mathbf{z})\right)^{1 / 2} .
$$

Proof. The combination of Theorem 4.7 and Lemma 4.4 leads to

$$
\begin{aligned}
\left\|\mathbf{u}-\mathbf{u}_{h}\right\|_{0} \lesssim & \lambda\left\|\mathbf{z}-\boldsymbol{I}_{h} \mathbf{z}\right\|_{s d}+\left(1+\eta^{3 / 2} \lambda\right)\left\|\mathbf{u}-\boldsymbol{I}_{h} \mathbf{u}\right\|_{0}+\lambda(1+\eta) \varepsilon(\mathbf{y}) \\
& +\lambda\left(\sum_{K \in \mathcal{T}_{h}} D_{K}^{2}\left(\boldsymbol{I}_{h} \mathbf{z}\right)+h_{K}^{2 l+1}\left\|\mathbf{z}^{v}\right\|_{l+1, K}^{2}\right)^{1 / 2}+\frac{1}{\alpha}\left(\sum_{K \in \mathcal{T}_{h}} D_{K}^{2}(\mathbf{z})\right)^{1 / 2} .
\end{aligned}
$$

By estimating

$$
\begin{aligned}
\sum_{K \in \mathcal{T}_{h}} D_{K}^{2}\left(\boldsymbol{I}_{h} \mathbf{z}\right) & \lesssim \sum_{K \in \mathcal{T}_{h}} D_{K}^{2}\left(\boldsymbol{I}_{h} \mathbf{z}-\mathbf{z}\right)+\sum_{K \in \mathcal{T}_{h}} D_{K}^{2}(\mathbf{z}) \\
& \lesssim \sum_{K \in \mathcal{T}_{h}}\|\mathbf{b}\|_{0, \infty ; K} h_{K}^{2 l+1}\left\|\mathbf{z}^{v}\right\|_{l+1, K}^{2}+h_{K}^{2 l+1}\left\|z^{p}\right\|_{l+1, K}^{2}+\sum_{K \in \mathcal{T}_{h}} D_{K}^{2}(\mathbf{z})
\end{aligned}
$$

we obtain the desired result.

\subsection{First optimize then discretize for SUPG/PSPG}

Now we first build up the optimality system on the continuous level and then discretize each equation separately. That means that we can choose an adequate stabilization term for the dual equation denoted $S_{h}^{z}$. This term contains the full residual of the dual equation and therefore depends on $\mathbf{z}$ and $\mathbf{u}$. The optimality system for the optimize-discretize- strategy is (3.6)-(3.8) with

$$
\begin{aligned}
S_{h}^{z}\left(\mathbf{z}_{h}, \mathbf{v}_{d}-\mathbf{v}_{h}\right)(\boldsymbol{\varphi})= & \sum_{K \in \mathcal{T}_{h}}\left\{\gamma_{K}\left(\nabla \cdot \mathbf{z}_{h}, \nabla \cdot \phi\right)_{K}+\delta_{K}\left(-\mu \Delta \mathbf{z}_{h}-(\mathbf{b} \cdot \nabla) \mathbf{z}_{h}+\sigma \mathbf{z}_{h}\right.\right. \\
& \left.\left.+\nabla \mathbf{z}_{h}^{p}+\mathbf{v}_{d}-\mathbf{v}_{h},-(\mathbf{b} \cdot \nabla) \phi+\nabla \xi\right)_{K}\right\} .
\end{aligned}
$$

Theorem 4.9. Let $(\mathbf{y}, \mathbf{u}, \mathbf{z})$ be the solution of (2.2)-(2.4) with control $\mathbf{u} \in H^{m+1}(\Omega)^{d}$ and $\left(\mathbf{y}_{h}, \mathbf{u}_{h}, \mathbf{z}_{h}\right)$ the solution of (3.6)-(3.8), then it holds with the parameter choice of (3.9) and the regularity assumption (4.2) the following estimate of optimal order:

$$
\left\|\mathbf{u}-\mathbf{u}_{h}\right\|_{0} \lesssim\left(1+\frac{\eta^{2}}{\alpha}\right)\left(\left(\sum_{K \in \mathcal{T}_{h}} h_{K}^{2(m+1)}\|\mathbf{u}\|_{m+1, K}^{2}\right)^{1 / 2}+\frac{\eta^{3 / 2}}{\alpha} \varepsilon\left(\mathbf{y}\left(\mathbf{u}_{h}\right)\right)+\frac{\eta^{1 / 2}}{\alpha} \varepsilon\left(\mathbf{z}\left(\mathbf{y}\left(\mathbf{u}_{h}\right)\right)\right)\right)
$$

Proof. Let $\left(\mathbf{y}\left(\mathbf{u}_{h}\right), \mathbf{z}\left(\mathbf{y}\left(\mathbf{u}_{h}\right)\right)\right)$ be the solution of the following equations

$$
\begin{aligned}
A\left(\mathbf{y}\left(\mathbf{u}_{h}\right), \boldsymbol{\varphi}\right)+B\left(\mathbf{u}_{h}, \boldsymbol{\phi}\right) & =\langle\mathbf{f}, \boldsymbol{\phi}\rangle & & \forall \boldsymbol{\varphi}=(\boldsymbol{\phi}, \xi) \in X, \\
A\left(\boldsymbol{\psi}, \mathbf{z}\left(\mathbf{y}\left(\mathbf{u}_{h}\right)\right)\right)-\left(\mathbf{v}\left(\mathbf{u}_{h}\right), \boldsymbol{\psi}^{v}\right) & =\left(-\mathbf{v}_{d}, \boldsymbol{\psi}^{v}\right) & & \forall \boldsymbol{\psi}=\left(\boldsymbol{\psi}^{v}, \psi^{p}\right) \in X .
\end{aligned}
$$


For these solutions and $\mathbf{z}$ of $(2.2)-(2.4)$ and $\mathbf{z}\left(\mathbf{u}_{h}\right):=\mathbf{z}\left(\mathbf{y}\left(\mathbf{u}_{h}\right)\right)$ it holds:

$$
\begin{aligned}
j^{\prime}(\mathbf{u})(\boldsymbol{\lambda}) & =-\alpha(\mathbf{u}, \boldsymbol{\lambda})+B\left(\boldsymbol{\lambda}, \mathbf{z}^{v}\right) & & \forall \boldsymbol{\lambda} \in Q, \\
j^{\prime}\left(\mathbf{u}_{h}\right)\left(\boldsymbol{\lambda}_{h}\right) & =-\alpha\left(\mathbf{u}_{h}, \boldsymbol{\lambda}_{h}\right)+B\left(\boldsymbol{\lambda}_{h}, \mathbf{z}^{v}\left(\mathbf{u}_{h}\right)\right) & & \forall \boldsymbol{\lambda}_{h} \in Q_{h} .
\end{aligned}
$$

Due to Lemma 3.1, and due the identities

$$
\begin{aligned}
-\left(\alpha \mathbf{u}, \mathbf{u}-\mathbf{u}_{h}\right)+B\left(\mathbf{u}-\mathbf{u}_{h}, \mathbf{z}^{v}\right) & =0=-\left(\alpha \mathbf{u}, \mathbf{u}-\boldsymbol{I}_{h} \mathbf{u}\right)+B\left(\mathbf{u}-\boldsymbol{I}_{h} \mathbf{u}, \mathbf{z}^{v}\right) \\
-\left(\alpha \mathbf{u}_{h}, \mathbf{u}_{h}-\boldsymbol{I}_{h} \mathbf{u}\right)+B\left(\mathbf{u}_{h}-\boldsymbol{I}_{h} \mathbf{u}, \mathbf{z}_{h}^{v}\right) & =0
\end{aligned}
$$

we get

$$
\begin{aligned}
\alpha\left\|\mathbf{u}-\mathbf{u}_{h}\right\|_{0}^{2} \leq & j^{\prime}(\mathbf{u})\left(\mathbf{u}-\mathbf{u}_{h}\right)-j^{\prime}\left(\mathbf{u}_{h}\right)\left(\mathbf{u}-\mathbf{u}_{h}\right) \\
= & -\left(\alpha \mathbf{u}, \mathbf{u}-\mathbf{u}_{h}\right)+B\left(\mathbf{u}-\mathbf{u}_{h}, \mathbf{z}^{v}\right)+\left(\alpha \mathbf{u}_{h}, \mathbf{u}-\mathbf{u}_{h}\right)-B\left(\mathbf{u}-\mathbf{u}_{h}, \mathbf{z}^{v}\left(\mathbf{u}_{h}\right)\right) \\
= & \left(\alpha \mathbf{u}_{h}, \mathbf{u}-\boldsymbol{I}_{h} \mathbf{u}\right)+B\left(\boldsymbol{I}_{h} u-\mathbf{u}, \mathbf{z}_{h}^{v}\right)+\left(\alpha \mathbf{u}, \boldsymbol{I}_{h} \mathbf{u}-\mathbf{u}\right)-B\left(\boldsymbol{I}_{h} \mathbf{u}-\mathbf{u}, \mathbf{z}^{v}\right) \\
& +B\left(\mathbf{u}_{h}-\mathbf{u}, \mathbf{z}^{v}\left(\mathbf{u}_{h}\right)-\mathbf{z}_{h}^{v}\right) \\
= & \alpha\left(\mathbf{u}-\mathbf{u}_{h}, \boldsymbol{I}_{h} \mathbf{u}-\mathbf{u}\right)+B\left(\boldsymbol{I}_{h} \mathbf{u}-\mathbf{u}, \mathbf{z}_{h}^{v}-\mathbf{z}^{v}\left(\mathbf{u}_{h}\right)\right)+B\left(\boldsymbol{I}_{h} \mathbf{u}-\mathbf{u}, \mathbf{z}^{v}\left(\mathbf{u}_{h}\right)-\mathbf{z}^{v}\right) \\
& +B\left(\mathbf{u}_{h}-\mathbf{u}, \mathbf{z}^{v}\left(\mathbf{u}_{h}\right)-\mathbf{z}_{h}^{v}\right) \\
\lesssim & \left\|\mathbf{z}_{h}^{v}-\mathbf{z}^{v}\left(\mathbf{u}_{h}\right)\right\|_{0}\left\|\boldsymbol{I}_{h} \mathbf{u}-\mathbf{u}\right\|_{0}+\left\|\mathbf{z}^{v}\left(\mathbf{u}_{h}\right)-\mathbf{z}^{v}\right\|_{0}\left\|\boldsymbol{I}_{h} \mathbf{u}-\mathbf{u}\right\|_{0}+\left\|\mathbf{z}^{v}\left(\mathbf{u}_{h}\right)-\mathbf{z}_{h}^{v}\right\|_{0}\left\|\mathbf{u}_{h}-\mathbf{u}\right\|_{0} \\
& +\alpha\left\|\mathbf{u}_{h}-\mathbf{u}\right\|_{0}\left\|\boldsymbol{I}_{h} \mathbf{u}-\mathbf{u}\right\|_{0} .
\end{aligned}
$$

By Young's inequality and due to $\left\|\mathbf{z}^{v}\left(\mathbf{u}_{h}\right)-\mathbf{z}^{v}\right\|_{0} \leq \eta\left\|\mathbf{y}\left(\mathbf{u}_{h}\right)-\mathbf{y}(\mathbf{u})\right\|_{0} \leq \eta^{2}\left\|\mathbf{u}-\mathbf{u}_{h}\right\|_{0}$ we obtain for arbitrary $\epsilon>0$

$$
\begin{aligned}
\alpha\left\|\mathbf{u}-\mathbf{u}_{h}\right\|_{0}^{2} & \lesssim \epsilon^{-1} \alpha\left\|\mathbf{u}-\boldsymbol{I}_{h} u\right\|_{0}^{2}+(2 \epsilon)^{-1}\left\|\mathbf{z}_{h}^{v}-\mathbf{z}^{v}\left(\mathbf{u}_{h}\right)\right\|_{0}^{2}+\frac{\epsilon}{\alpha}\left\|\mathbf{z}^{v}\left(\mathbf{u}_{h}\right)-\mathbf{z}^{v}\right\|_{0}^{2}+2 \epsilon \alpha\left\|\mathbf{u}_{h}-\mathbf{u}\right\|_{0}^{2} \\
& \leq \epsilon^{-1} \alpha\left\|\mathbf{u}-\boldsymbol{I}_{h} \mathbf{u}\right\|_{0}^{2}+(2 \epsilon)^{-1}\left\|\mathbf{z}_{h}^{v}-\mathbf{z}^{v}\left(\mathbf{u}_{h}\right)\right\|_{0}^{2}+\epsilon\left(2 \alpha+(\sigma+\mu)^{-1} \alpha^{-1}\right)\left\|\mathbf{u}_{h}-\mathbf{u}\right\|_{0}^{2}
\end{aligned}
$$

With the choice $\epsilon:=\frac{\alpha}{2}\left(2 \alpha+\eta^{2} \alpha^{-1}\right)^{-1}$ we end up with

$$
\frac{\alpha}{2}\left\|\mathbf{u}-\mathbf{u}_{h}\right\|_{0}^{2} \lesssim \epsilon^{-1} \alpha\left\|\mathbf{u}-\boldsymbol{I}_{h} \mathbf{u}\right\|_{0}^{2}+\epsilon^{-1}\left\|\mathbf{z}_{h}^{v}-\mathbf{z}^{v}\left(\mathbf{u}_{h}\right)\right\|_{0}^{2} .
$$

Due to $\epsilon^{-1} \lesssim 1+\eta^{2} \alpha^{-1}$, we obtain

$$
\left\|\mathbf{u}-\mathbf{u}_{h}\right\|_{0}^{2} \lesssim\left(1+\frac{\eta^{2}}{\alpha}\right)\left(\sum_{K \in \mathcal{T}_{h}} h_{K}^{2(m+1)}\|\mathbf{u}\|_{m+1, K}^{2}+\frac{1}{\alpha}\left\|\mathbf{z}_{h}^{v}-\mathbf{z}^{v}\left(\mathbf{u}_{h}\right)\right\|_{0}^{2}\right) .
$$

In order to bound the last term, we introduce the continuous adjoint solution $\mathbf{z}\left(\mathbf{y}_{h}\left(\mathbf{u}_{h}\right)\right)$ which is the solution of

$$
A\left(\boldsymbol{\psi}, \mathbf{z}\left(\mathbf{y}_{h}\left(\mathbf{u}_{h}\right)\right)\right)=\left(\mathbf{v}_{h}-\mathbf{v}_{d}, \boldsymbol{\psi}^{v}\right) \quad \forall \boldsymbol{\psi}=\left(\boldsymbol{\psi}^{v}, \psi^{p}\right) \in X .
$$

Due to the stability of the continuous problem and due to a standard estimate a priori estimates of adjoint PSPG/SUPG stabilization it holds:

$$
\begin{aligned}
\left\|\mathbf{z}_{h}^{v}-\mathbf{z}^{v}\left(\mathbf{u}_{h}\right)\right\|_{0} & \leq\left\|\mathbf{z}_{h}^{v}-\mathbf{z}^{v}\left(\mathbf{y}_{h}\left(\mathbf{u}_{h}\right)\right)\right\|_{0}+\left\|\mathbf{z}^{v}\left(\mathbf{y}_{h}\left(\mathbf{u}_{h}\right)\right)-\mathbf{z}^{v}\left(\mathbf{u}_{h}\right)\right\|_{0} \\
& \lesssim \eta^{1 / 2}\left\|\mathbf{z}_{h}-\mathbf{z}\left(\mathbf{y}_{h}\left(\mathbf{u}_{h}\right)\right)\right\|_{s d}+\eta^{3 / 2}\left\|\mathbf{y}_{h}\left(\mathbf{u}_{h}\right)-\mathbf{y}\left(\mathbf{u}_{h}\right)\right\|_{s d} .
\end{aligned}
$$

By help of a priori standard estimates for the primal error $\left\|\mathbf{y}_{h}\left(\mathbf{u}_{h}\right)-\mathbf{y}\left(\mathbf{u}_{h}\right)\right\|_{s d}$ and dual error $\| \mathbf{z}_{h}\left(\mathbf{y}_{h}\left(\mathbf{u}_{h}\right)\right)-$ $\mathbf{z}\left(\mathbf{y}_{h}\left(\mathbf{u}_{h}\right)\right) \|_{s d}$ we arrive at the desired estimate. 


\subsection{A priori estimate for local projection stabilization}

For an a priori analysis we refer to [4] where symmetric stabilization methods for optimal control problems with the Oseen system are analyzed. The local projection stabilization is a particular technique which fits into that framework. There is no difference in optimize-discretize or discretize-optimize, because the resulting systems are completely identical:

$$
A_{h}\left(\Psi, \mathbf{z}_{h}\right)=A\left(\Psi, \mathbf{z}_{h}\right)+S_{h}^{l p s}(\Psi, 0)\left(\mathbf{z}_{h}\right)
$$

and

$$
S_{h}^{l p s}(\Psi, 0)\left(\mathbf{z}_{h}\right)=S_{h}^{l p s}\left(\mathbf{z}_{h}, 0\right)(\Psi)=S_{h}^{l p s}\left(\mathbf{z}_{h}, \mathbf{v}_{d}-\mathbf{v}_{h}\right)(\Psi)=S_{h}^{l p s *}\left(\mathbf{z}_{h}, \mathbf{v}_{d}-\mathbf{v}_{h}\right)(\Psi) .
$$

We get the following result, see [4]:

Theorem 4.10. Let $(\mathbf{y}, \mathbf{u}, \mathbf{z}) \in X \times Q \times X$ be the solution of the continuous optimization problem (2.2)-(2.4) with regularity (4.2) and $\left(\mathbf{y}_{h}, \mathbf{u}_{h}, \mathbf{z}_{h}\right) \in X_{h} \times Q_{h} \times X_{h}$ be the solution of discrete optimization problem (3.3)-(3.5) with LPS stabilization (3.13). Then it holds

$$
\left\|\mathbf{u}-\mathbf{u}_{h}\right\|_{0}^{2} \lesssim \alpha^{-2} \eta(\varepsilon(\mathbf{y})+\varepsilon(\mathbf{z}))+\left(1+\alpha^{-2}\right) \sum_{K \in \mathcal{T}_{h}} h_{K}^{2 m+2}\|\mathbf{u}\|_{m+1, K}^{2} .
$$

\subsection{Discussion on the a priori results}

Remark 4.11. The a priori estimates for SUPG/PSPG in the optimize-discretize setting (Thm. 4.9) and the symmetric LPS method (Thm. 4.10) are both quasi-optimal in the sense that the $h$-depending convergence order scale is the same as for the pure primal problem without control, i.e. convergence order $h^{r+1 / 2}$ for convection dominated flows. For SUPG/PSPG in the discretize-optimize setting, the convergence order is worse. In particular with respect to the error in the adjoint state and according to Lemma 4.4, the order of convergence is limited to $h^{1 / 2}$ independently of the regularity of the solution and independently of the order of finite elements.

Remark 4.12. Furthermore, the case $\sigma \geq 1$ leads to $\eta \leq 1$ and $\lambda \lesssim \alpha^{-1 / 2}$. This implies that all a priori estimates stated above become (formally) independent of the parameters $\sigma$ and $\mu$. If additionally $\alpha \geq 1$, the estimates become also $\alpha$-independent, in particular,

- SUPG discretize-optimize:

$$
\left\|\mathbf{u}-\mathbf{u}_{h}\right\|_{0} \lesssim \varepsilon(\mathbf{y})+\varepsilon(\mathbf{z})+\left\|\mathbf{u}-\boldsymbol{I}_{h} \mathbf{u}\right\|+\left(\sum_{K \in \mathcal{T}_{h}} D_{K}^{2}(\mathbf{z})\right)^{1 / 2}
$$

- SUPG optimize-discretize:

$$
\left\|\mathbf{u}-\mathbf{u}_{h}\right\|_{0} \lesssim \varepsilon\left(\mathbf{y}\left(\mathbf{u}_{h}\right)\right)+\varepsilon\left(\mathbf{z}\left(\mathbf{y}\left(\mathbf{u}_{h}\right)\right)\right)+\left(\sum_{K \in \mathcal{T}_{h}} h_{K}^{2(m+1)}\|\mathbf{u}\|_{m+1, K}^{2}\right)^{1 / 2} ;
$$

- LPS:

$$
\left\|\mathbf{u}-\mathbf{u}_{h}\right\|_{0} \lesssim \varepsilon(\mathbf{y})+\varepsilon(\mathbf{z})+\left(\sum_{K \in \mathcal{T}_{h}} h_{K}^{2 m+2}\|\mathbf{u}\|_{m+1, K}^{2}\right)^{1 / 2}
$$




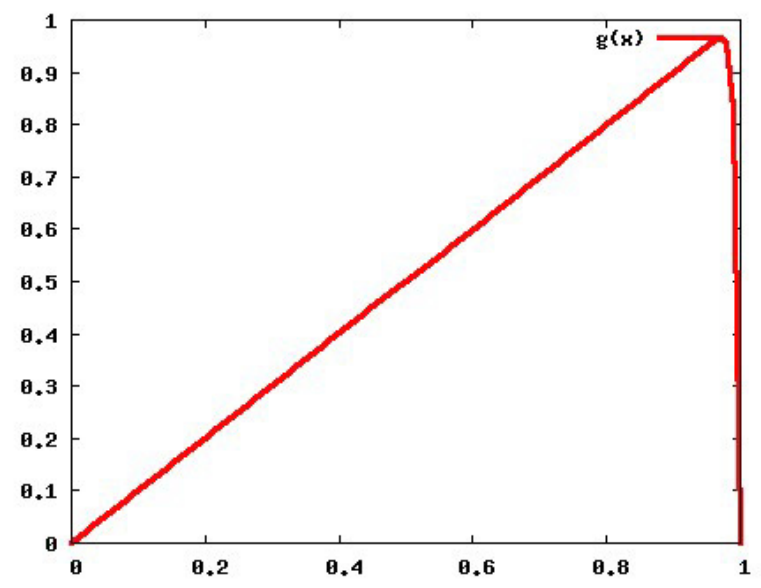

Figure 1. The function $g$ (right).

\section{NumeriCAL EXPERIMENT}

In order to validate the analytical results presented so far, we consider a model problem with analytical solution. We are interested in particular in the decline of convergence order for SUPG/PSPG in the setting discretize-optimize (DO). The domain is the two dimensional unit square $\Omega=(0,1)^{2}$ with the boundaries $\Gamma_{S}, \Gamma_{E}, \Gamma_{N}, \Gamma_{W}$, where the indices refer to the cardinal points, e.g. S(outh) etc. The boundary conditions are given by:

$$
\begin{aligned}
& \mathbf{v}^{1}=0 ; \quad \mu\left(\nabla \mathbf{v}^{2}, \mathbf{n}\right)-p \mathbf{n}^{2}=0 \quad \text { on } \Gamma_{S} \cup \Gamma_{N} \\
& \mathbf{v}^{2}=0 ; \quad \mu\left(\nabla \mathbf{v}^{1}, \mathbf{n}\right)-p \mathbf{n}^{1}=0 \quad \text { on } \Gamma_{E} \cup \Gamma_{W} \text {. }
\end{aligned}
$$

The exact solution $\mathbf{y}=(\mathbf{v}, p)$ reads:

$$
\mathbf{v}^{1}(x, y)=g(y), \mathbf{v}^{2}(x, y)=g(x), \quad p=0,
$$

with the viscosity-depending function (see Fig. 1)

$$
g(x):=x-\frac{1-\mathrm{e}^{x / \mu}}{1-\mathrm{e}^{1 / \mu}} .
$$

The adjoint state $\mathbf{z}=\left(\mathbf{z}^{v}, z^{p}\right)$ and the control are given by

$$
\mathbf{z}^{v, 1}(x, y)=g(1-y), \quad \mathbf{z}^{v, 2}(x, y)=g(1-x), \quad \mathbf{z}^{p}=0 \quad \mathbf{u}=-\mathbf{z}^{v} .
$$

The viscosity is set to $\mu=7.5 \times 10^{-3}$ in order to enforce a sharp boundary layer. The constants in the stabilization parameters are always chosen as $\gamma_{0}=\delta_{0}=0.2$, and the regularization parameter of the cost fuctional is $\alpha=1$.

In Figure 2 we show the discrete control $\mathbf{u}_{h}$ with SUPG/PSPG in the combination optimize-discretize (OD) and discretize-optimize (DO) with Q2 elements. The Q2 solution with DO exhibit strong oscillations. It is remarkable that these spurious modes are not even restricted at the neighborhood of the boundary layer. The non-consistent stabilization leads obviously to parasitic currents in the entire domain.

In Table 1 we list the order of convergence with respect to the state, the adjoint state, the control and the error in the functional for the DO and the OD approach using SUPG/PSPG - stabilization and $Q 1 / Q 2$ elements. For $Q 1$ elements, there is no significant difference and all quantities show a convergence rate of about 

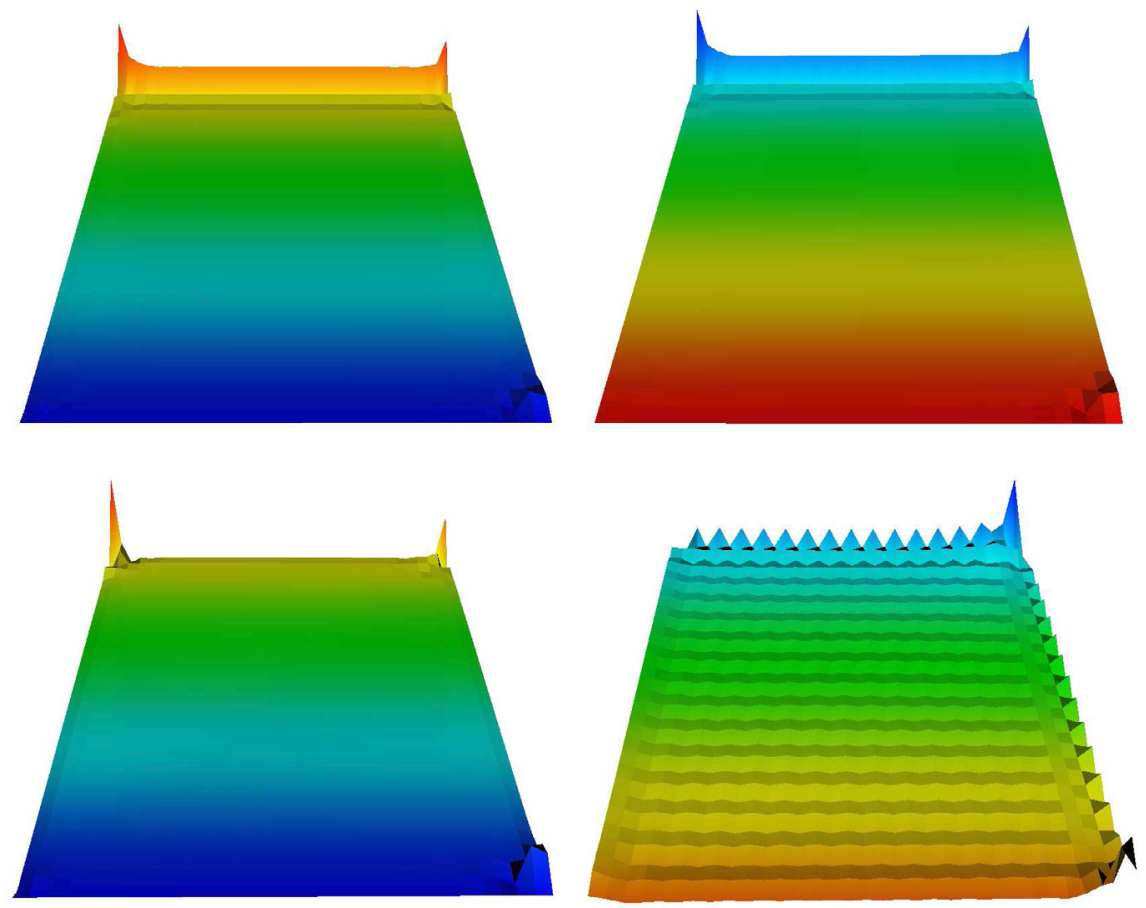

Figure 2. Q2 solutions with SUPG/PSPG. State $\mathbf{v}_{h}^{1}$ OD (upper left), control $\mathbf{u}_{h}^{1}$ OD (upper right), state $\mathbf{v}_{h}^{1}$ DO (lower left) and control $\mathbf{u}_{h}^{1}$ DO (lower right).

$3 / 2$ in the $L^{2}$-norm. In case of higher order $(Q 2)$ elements this holds also true for the error in state variable. However, the error in the adjoint solution for the DO setting is even larger than for $Q 1$ elements. This lack of accuracy carries over to the error in the control. For OD we obtain a $L^{2}$ convergence rate of approximately $5 / 2$ in all variables.

In Table 2 the convergence rates of all quantities using LPS-stabilization are listed. The optimal orders are reached quite earlier than in case of SUPG/PSPG-stabilization. Comparing the error $j(\mathbf{u})-j_{h}\left(\mathbf{u}_{h}\right)$ for SUPG/PSPG in the DO setting and for LPS, the error is smaller by more than three magnitudes on the same mesh (the finest one) and the same finite element (bi-quadratics) when a symmetric stabilization is chosen. We think that this is a also remarkable result.

\section{Summary}

We have analyzed two types of stabilized finite element techniques for optimal control problems with the Oseen system, namely the local projection stabilization (LPS) and the combination of streamline upwind PetrovGalerkin (SUPG) and pressure stabilized Petrov-Galerkin (PSPG). There is a fundamental difference between these two methods in the context of optimize-discretize (OD) and discretize-optimize (DO). Whereas OD and DO are identical for LPS due to its symmetry properties, the ordering of discretization and optimization leads to different schemes for SUPG/PSPG. We have proved that LPS and OD with SUPG/PSPG lead to optimal order of convergence in the control. In contrast to this, DO with SUPG/PSPG gives only a suboptimal convergence order. The reason is the inconsistent discretization of the adjoint equation. In a numerical test problem with a sharp boundary layer we investigated whether this decrease in convergence order is observable in practice. It turns out that for low order finite elements (Q1), no substantial difference is observed. However, DO with SUPG/PSPG for higher order elements as e.g. Q2, leads to a clear decline of convergence order in the control 
TABLE 1. Errors and convergence orders obtained with SUPG/PSPG.

\begin{tabular}{|c|c|c|c|c|c|c|c|c|c|c|c|c|}
\hline \multirow{2}{*}{$\begin{array}{l}h= \\
2^{-l}\end{array}$} & \multicolumn{4}{|c|}{$\mid \mathbf{y}-\mathbf{y}_{h}$} & \multicolumn{4}{|c|}{$\mid \mathbf{z}-\mathbf{z}_{h}$} & \multicolumn{2}{|c|}{$\mathbf{u}-\mathbf{u}_{h}$} & \multicolumn{2}{|c|}{$j(\mathbf{u})-j_{h}\left(\mathbf{u}_{h}\right)$} \\
\hline & $\|\cdot\|_{0}$ & Order & $\|\cdot\| \cdot \|_{s d}$ & Order & $\cdot \|_{0}$ & Order & $\cdot\|\|_{s d}$ & Order & $\cdot \|_{0}$ & Order & Value & Order \\
\hline \multicolumn{13}{|c|}{ SUPG/PSPG Q1 optimize-discretize } \\
\hline 3 & $2.65 \mathrm{e}-1$ & & $9.58 \mathrm{e}-1$ & & $2.68 \mathrm{e}-1$ & & $9.76 \mathrm{e}-1$ & & $1.89 \mathrm{e}-1$ & & $2.91 \mathrm{e}-2$ & \\
\hline 4 & $1.50 \mathrm{e}-1$ & 0.82 & $7.77 \mathrm{e}-1$ & 0.30 & $1.50 \mathrm{e}-1$ & 0.83 & $7.77 \mathrm{e}-1$ & 0.33 & $1.06 \mathrm{e}-1$ & 0.83 & $2.06 \mathrm{e}-2$ & 0.50 \\
\hline 5 & $6.62 \mathrm{e}-2$ & 1.18 & $7.44 \mathrm{e}-1$ & 0.06 & $6.62 \mathrm{e}-2$ & 1.18 & $7.43 \mathrm{e}-1$ & 0.06 & $4.68 \mathrm{e}-2$ & 1.18 & $1.03 \mathrm{e}-2$ & 1.00 \\
\hline 6 & $2.42 \mathrm{e}-2$ & 1.45 & $5.30 \mathrm{e}-1$ & 0.49 & $2.42 \mathrm{e}-2$ & 1.45 & $5.29 \mathrm{e}-1$ & 0.49 & $1.71 \mathrm{e}-2$ & 1.45 & $4.24 \mathrm{e}-3$ & 1.28 \\
\hline 7 & $8.15 \mathrm{e}-3$ & 1.57 & $2.96 \mathrm{e}-1$ & 0.84 & $8.15 \mathrm{e}-3$ & 1.57 & $2.96 \mathrm{e}-1$ & 0.84 & $5.76 \mathrm{e}-3$ & 1.57 & $1.59 \mathrm{e}-3$ & 1.42 \\
\hline \multicolumn{13}{|c|}{ SUPG/PSPG Q1 discretize-optimize } \\
\hline 3 & $2.67 \mathrm{e}-1$ & & $9.55 \mathrm{e}-1$ & & $2.70 \mathrm{e}-1$ & & $9.70 \mathrm{e}-1$ & & $1.52 \mathrm{e}-1$ & & $4.33 \mathrm{e}-2$ & \\
\hline 4 & $1.50 \mathrm{e}-1$ & 0.83 & $7.77 \mathrm{e}-1$ & 0.30 & $1.51 \mathrm{e}-1$ & 0.84 & $7.78 \mathrm{e}-1$ & 0.32 & $7.85 \mathrm{e}-2$ & 0.95 & $2.53 \mathrm{e}-2$ & 0.78 \\
\hline 5 & $6.61 \mathrm{e}-2$ & 1.19 & $7.44 \mathrm{e}-1$ & 0.06 & $6.65 \mathrm{e}-2$ & 1.19 & $7.44 \mathrm{e}-1$ & 0.06 & $2.86 \mathrm{e}-2$ & 1.46 & $1.17 \mathrm{e}-2$ & 1.12 \\
\hline 6 & $2.42 \mathrm{e}-2$ & 1.45 & $5.30 \mathrm{e}-1$ & 0.49 & $2.43 \mathrm{e}-2$ & 1.45 & $5.30 \mathrm{e}-1$ & 0.49 & $6.62 \mathrm{e}-3$ & 2.11 & $4.58 \mathrm{e}-3$ & 1.35 \\
\hline 7 & $8.11 \mathrm{e}-3$ & 1.58 & $2.96 \mathrm{e}-1$ & 0.84 & $8.16 \mathrm{e}-3$ & 1.57 & $2.96 \mathrm{e}-1$ & 0.84 & $1.52 \mathrm{e}-3$ & 2.12 & $1.65 \mathrm{e}-3$ & 1.47 \\
\hline \multicolumn{13}{|c|}{ SUPG/PSPG Q2 optimize-discretize } \\
\hline 3 & $1.91 \mathrm{e}-1$ & & $7.52 \mathrm{e}-1$ & & $1.89 \mathrm{e}-1$ & & $7.47 \mathrm{e}-1$ & & $1.34 \mathrm{e}-1$ & & $6.59 \mathrm{e}-2$ & \\
\hline 4 & $9.86 \mathrm{e}-2$ & 0.96 & $9.67 \mathrm{e}-1$ & -0.36 & $9.77 \mathrm{e}-2$ & 0.95 & $9.66 \mathrm{e}-1$ & -0.37 & $6.91 \mathrm{e}-2$ & 0.95 & $3.22 \mathrm{e}-2$ & 1.03 \\
\hline 5 & $4.05 \mathrm{e}-2$ & 1.29 & $6.27 \mathrm{e}-1$ & 0.63 & $4.00 \mathrm{e}-2$ & 1.29 & $6.26 \mathrm{e}-1$ & 0.63 & $2.83 \mathrm{e}-2$ & 1.29 & $1.17 \mathrm{e}-2$ & 1.26 \\
\hline 6 & $1.06 \mathrm{e}-2$ & 1.93 & $2.32 \mathrm{e}-1$ & 1.44 & $1.03 \mathrm{e}-2$ & 1.95 & $2.31 \mathrm{e}-1$ & 1.44 & $7.32 \mathrm{e}-3$ & 1.95 & $2.62 \mathrm{e}-3$ & 2.16 \\
\hline 7 & $1.69 \mathrm{e}-3$ & 2.65 & $5.68 \mathrm{e}-2$ & 2.03 & $1.55 \mathrm{e}-3$ & 2.74 & $5.68 \mathrm{e}-2$ & 2.03 & $1.09 \mathrm{e}-3$ & 2.75 & $2.54 \mathrm{e}-4$ & 3.37 \\
\hline \multicolumn{13}{|c|}{ SUPG/PSPG Q2 discretize-optimize } \\
\hline 3 & $1.89 \mathrm{e}-1$ & & $7.50 \mathrm{e}-1$ & & $2.20 \mathrm{e}-1$ & & $1.68 \mathrm{e}+0$ & & $9.01 \mathrm{e}-2$ & & $8.05 \mathrm{e}-2$ & \\
\hline 4 & $9.74 \mathrm{e}-2$ & 0.95 & $9.66 \mathrm{e}-1$ & -0.37 & $1.29 \mathrm{e}-1$ & 0.77 & $1.62 \mathrm{e}+0$ & 0.05 & $4.49 \mathrm{e}-2$ & 1.01 & $3.83 \mathrm{e}-2$ & 1.07 \\
\hline 5 & $4.01 \mathrm{e}-2$ & 1.28 & $6.26 \mathrm{e}-1$ & 0.63 & $7.54 \mathrm{e}-2$ & 0.78 & $1.52 \mathrm{e}+0$ & 0.09 & $2.59 \mathrm{e}-2$ & 0.80 & $1.35 \mathrm{e}-2$ & 1.50 \\
\hline 6 & $1.05 \mathrm{e}-2$ & 1.93 & $2.32 \mathrm{e}-1$ & 1.44 & $3.76 \mathrm{e}-2$ & 1.00 & $1.20 \mathrm{e}+0$ & 0.34 & $1.31 \mathrm{e}-2$ & 0.98 & $3.00 \mathrm{e}-3$ & 2.17 \\
\hline 7 & $1.66 \mathrm{e}-3$ & 2.66 & $5.58 \mathrm{e}-2$ & 2.03 & $1.48 \mathrm{e}-2$ & 1.35 & $7.86 \mathrm{e}-1$ & 0.62 & $5.27 \mathrm{e}-3$ & 1.32 & $3.18 \mathrm{e}-4$ & 3.24 \\
\hline
\end{tabular}

TABLE 2. Errors and convergence orders obtained with LPS.

\begin{tabular}{|c|c|c|c|c|c|c|c|c|}
\hline \multirow{2}{*}{$\begin{array}{l}h= \\
2^{-l}\end{array}$} & \multicolumn{2}{|c|}{$\left|\mathbf{y}-\mathbf{y}_{h}\right|$} & \multicolumn{2}{|c|}{$\mathbf{z}-\mathbf{z}_{h}$} & \multicolumn{2}{|c|}{$\mathbf{u}-\mathbf{u}_{h}$} & \multicolumn{2}{|c|}{$j(\mathbf{u})-j_{h}\left(\mathbf{u}_{h}\right)$} \\
\hline & 0 & Order & 0 & Order & $\cdot \|_{0}$ & Order & Value & Order \\
\hline \multicolumn{9}{|c|}{ LPS Q1 } \\
\hline 3 & $2.70 \mathrm{e}-1$ & & $2.77 \mathrm{e}-1$ & & $1.96 \mathrm{e}-1$ & & $4.80 \mathrm{e}-3$ & \\
\hline 4 & $1.58 \mathrm{e}-1$ & 0.77 & $1.60 \mathrm{e}-1$ & 0.79 & $1.13 \mathrm{e}-1$ & 0.79 & $3.03 \mathrm{e}-3$ & 0.66 \\
\hline 5 & $7.20 \mathrm{e}-2$ & 1.14 & $7.25 \mathrm{e}-2$ & 1.15 & $5.12 \mathrm{e}-2$ & 1.15 & $1.06 \mathrm{e}-3$ & 1.52 \\
\hline 6 & $2.48 \mathrm{e}-2$ & 1.54 & $2.48 \mathrm{e}-2$ & 1.54 & $1.76 \mathrm{e}-2$ & 1.54 & $1.62 \mathrm{e}-4$ & 2.71 \\
\hline 7 & $6.46 \mathrm{e}-3$ & 1.94 & $6.48 \mathrm{e}-3$ & 1.94 & $4.58 \mathrm{e}-3$ & 1.94 & $1.60 \mathrm{e}-5$ & 3.35 \\
\hline \multicolumn{9}{|c|}{ LPS Q2 } \\
\hline 3 & $2.00 \mathrm{e}-1$ & & $2.04 \mathrm{e}-1$ & & $1.44 \mathrm{e}-1$ & & $1.80 \mathrm{e}-3$ & \\
\hline 4 & $9.24 \mathrm{e}-2$ & 1.12 & $9.31 \mathrm{e}-2$ & 1.13 & $6.58 \mathrm{e}-2$ & 1.13 & $1.13 \mathrm{e}-3$ & 0.67 \\
\hline 5 & $3.08 \mathrm{e}-2$ & 1.59 & $3.09 \mathrm{e}-2$ & 1.59 & $2.18 \mathrm{e}-2$ & 1.59 & $1.35 \mathrm{e}-4$ & 3.06 \\
\hline 6 & $6.28 \mathrm{e}-3$ & 2.29 & $6.29 \mathrm{e}-3$ & 2.30 & $4.44 \mathrm{e}-3$ & 2.30 & $6.72 \mathrm{e}-6$ & 4.33 \\
\hline 7 & $7.80 \mathrm{e}-4$ & 3.01 & $7.81 \mathrm{e}-4$ & 3.01 & $5.53 \mathrm{e}-4$ & 3.01 & $2.28 \mathrm{e}-7$ & 4.88 \\
\hline
\end{tabular}

and in the adjoint variable. LPS and OD with SUPG/PSPG remain optimal for Q2 elements. However, the symmetric method shows by far the best performance in terms of accuracy.

Acknowledgements. This work is partially supported by the DFG Priority Program SPP 1253 (Optimization with PDE). This support is gratefully acknowledged. 


\section{REFERENCES}

[1] F. Abraham, M. Behr and M. Heinkenschloss, The effect of stabilization in finite element methods for the optimal boundary control of the Oseen equations. Finite Elem. Anal. Des. 41 (2004) 229-251.

[2] R. Becker and M. Braack, A two-level stabilization scheme for the Navier-Stokes equations, in Numerical Mathematics and Advanced Applications, ENUMATH 2003. edited by, M. Feistauer et al., Springer (2004) 123-130.

[3] R. Becker and B. Vexler, Optimal control of the convection-diffusion equation using stabilized finite element methods. Numer. Math. 106 (2007) 349-367.

[4] M. Braack, Optimal control in fluid mechanics by finite elements with symmetric stabilization. SIAM J. Control Optim. 48 (2009) 672-687.

[5] M. Braack and E. Burman, Local projection stabilization for the Oseen problem and its interpretation as a variational multiscale method. SIAM J. Numer. Anal. 43 (2006) 2544-2566.

[6] M. Braack, E. Burman, V. John and G. Lube, Stabilized finite element methods for the generalized Oseen problem. Comput. Methods Appl. Mech. Engrg. 196 (2007) 853-866.

[7] A.N. Brooks and T.J.R. Hughes, Streamline upwind Petrov-Galerkin formulation for convection dominated flows with particular emphasis on the incompressible Navier-Stokes equations. Comput. Methods Appl. Mech. Engrg. 32 (1982) 199-259.

[8] S.S. Collis and M. Heinkenschloss, Analysis of the streamline upwind/Petrov Galerkin method applied to the solution of optimal control problems. Technical report 02-01, Rice University, Houston, TX (2002).

[9] L. Dedé and A. Quarteroni, Optimal control and numercal adaptivity for advection-diffusion equations. ESIAM: M2AN 39 (2005) 1019-1040.

[10] V. Girault and P.-A. Raviart, Finite Elements for the Navier Stokes Equations. Springer, Berlin (1986).

[11] M. Heinkenschloss and D. Leykekhman, Local error estimates for SUPG solutions of advection-dominated elliptic linearquadratic optimal control problems. SIAM J. Numer. Anal. 47 (2010) 4607-4638.

[12] M. Hinze, N. Yan and Z. Zhou, Variational discretization for optimal control governed by convection dominated diffusion equations. J. Comput. Math. 27 (2009) 237-253.

[13] C. Johnson and J. Saranen, Streamline diffusion methods for the incompressible Euler and Navier-Stokes equations. Math. Comput. 47 (1986) 1-18.

[14] G. Lube and G. Rapin, Residual-based stabilized higher-order FEM for a generalized Oseen problem. Math. Models Methods Appl. Sci. 16 (2006) 949-966.

[15] G. Lube and G. Rapin, Residual-based stabilized higher-order FEM for a generalized Oseen problem. Math. Models Methods Appl. Sci. 16 (2006) 949-966.

[16] G. Lube and B. Tews, Optimal control of singularly perturb advection-diffusion-reaction problems. Math. Models Appl. Sci. 20 (2010) 1-21.

[17] G. Matthies, P. Skrzypacz and L. Tobiska, A unified convergence analysis for local projection stabilisations applied ro the Oseen problem. ESAIM: M2AN 41 (2007) 713-742.

[18] N. Yan and Z. Zhou, A priori and a posteriori error estimates of streamline diffusion finite element method for optimal control problems governed by convection dominated diffusion equation. NMTMA 1 (2008) 297-320.

[19] N. Yan and Z. Zhou, A priori and a posteriori error analysis of edge stabilization Galerkin method for the optimal control problem governed by convection dominated diffusion equation. J. Comput. Appl. Math. 223 (2009) 198-217. 